\title{
Current noise in molecular junctions: effects of the electron-phonon interaction
}

\author{
Federica Haupt,, * Tomáš Novotný, ${ }^{2, \oplus}$ and Wolfgang Belzig1, $¥$ \\ ${ }^{1}$ Fachbereich Physik, Universität Konstanz, D- 78457 Konstanz, Germany \\ ${ }^{2}$ Department of Condensed Matter Physics, Faculty of Mathematics and Physics, \\ Charles University in Prague, Ke Karlovu 5, CZ-121 16 Praha 2, Czech Republic
}

(Dated: August 3, 2018)

\begin{abstract}
We study inelastic effects on the electronic current noise in molecular junctions, due to the coupling between transport electrons and vibrational degrees of freedom. Using a full counting statistics approach based on the generalized Keldysh Green's function technique, we calculate in an unified manner both the mean current and the zero-frequency current noise. For multilevel junctions with weak electron-phonon coupling, we give analytical formulas for the lowest order inelastic corrections to the noise in terms of universal temperature- and voltage-dependent functions and junctiondependent prefactors, which can be evaluated microscopically, e.g. with ab-initio methodologies. We identify distinct terms corresponding to the mean-field contribution to noise and to the vertex corrections, and we show that the latter contribute substantially to the inelastic noise. Finally, we illustrate our results by a simple model of two electronic levels which are mutually coupled by the electron-phonon interaction and show that the inelastic noise spectroscopy is a sensitive diagnostic tool.

PACS numbers: 72.70.+m, 72.10.Di, 85.65.+h, 73.63.-b
\end{abstract}

\section{INTRODUCTION}

Recent progress in experimental techniques, such as break junctions and scanning tunneling microscopy, allows to electrically contact single molecules, to create and manipulate atomic wires, and to investigate the electronic transport properties of these nanoscopic objects,$\frac{1,2}{1}$ Contrary to larger devices, atomic-scale ones usually retain their microscopic features, which are then observable in the transport spectroscopy. Apart from the purely electronic contributions, other degrees of freedom such as vibrational modes or local spins can be addressed and revealed by point-contact spectroscopy $\underline{\underline{3}}$ (PCS) or by inelastic electron tunneling spectroscopy (IETS) $\underline{\underline{4}}$ These techniques have been extensively used in the recent past to reveal inelastic features in the non-linear conductance due to vibrations $\stackrel{5-12}{\underline{-12}}$ or local spin excitations $\stackrel{13-15}{=}$ triggering an intense theoretical activity. So far most studies focused on the current-voltage characteristics, and present day PCS/IETS theories, $\underline{16}-\underline{-31}$ often based on abinitio calculations, allow to make detailed predictions for the conductance that compare favorably with experimental results.

Electronic current (shot) noise $\frac{32,33}{2}$ is another quantity of fundamental interest in transport, representing the second cumulant of the current distribution within the full counting statistics methodology $\underline{34}$ Although the measurement of higher order cumulants is experimentally challenging, shot noise in atomic contacts and molecular junctions has been already measured in the small voltage (elastic) regime, $\stackrel{35-37}{-37}$ and there are ongoing experimental efforts to address the inelastic noise signal as well. ${ }^{38}$ These progresses made the investigation of effects due to electron-phonon (e-ph) interaction on the current noise an appealing task from the theoretical point of view, with the ultimate goal of allowing for prediction for the noise in molecular junctions as reliable as those now available for the non-linear conductance.

Since the noise is technically represented by a twoparticle non-equilibrium correlation function, its direct evaluation poses a significant challenge compared to the mean current. For molecular junctions weakly coupled to leads, noise calculations based on one-level models have been put forward within the rate equation approach 39.40 In the opposite limit, pioneering works based on the nonequilibrium Green's functions formalism ${ }^{41,42}$ adopted a mean-field-like approximation for the noise, thus neglecting the contributions due to the vertex corrections. A very convenient tool to overcome these limitations is the full counting statistics $, 34,43$ since it allows to address the noise and other current cumulants, while taking consistently into account all contributions due to $e$-ph coupling up to a given order in perturbation theory. Simultaneously with other two groups $\stackrel{44.45}{\stackrel{4}{w}}$ we exploited such an approach to analyze the transport properties of a simple model for molecular junctions consisting of a single resonant level symmetrically coupled to metallic leads and weakly interacting with a single phonon mode $\stackrel{46}{\underline{4}}$ Despite its simplicity, this model can be applied to the experimentally relevant case of junctions formed by a single hydrogen/deuterium molecule between platinum leads, $\stackrel{7}{-}$ and in this case we predicted a significant inelastic contribution to the current noise $\underline{\underline{46}}$

In this paper we go beyond such a simple model and we extend our theory for inelastic noise ${ }^{46}$ to more complex molecular junctions and to atomic wires. In fact, we consider the case of a junction formed by multiple electronic levels with asymmetric coupling to leads, and derive analytical formulas for the corrections to current and noise due to weak electron-phonon coupling. We express our result in terms of universal temperature- and voltagedependent functions and junction-dependent prefactors. 
These expressions, when supplemented with ab-initio calculations to estimate microscopically the prefactors characterizing a given junction, can serve as a basis to make realistic predictions for the current noise in a relevant class of molecular and atomic-size junctions. In this respect, our work can be viewed as a direct extension of the corresponding lowest order expansion scheme developed for the non-linear conductance ${ }^{18-20}$ In addition, we identify the contributions to noise due to the vertex corrections and show that, even in limit of weak $e$-ph coupling, they need to be taken into account in order to obtain accurate results and to comply with the fluctuationdissipation theorem.

The paper is organized as follows. After a brief description of the model of a multi-level junction coupled to leads and weakly interacting with a number of vibronic modes (phonons) in Sec. II] we introduce the methodology of the noise calculation via extended Keldysh Green's functions in Sec. III. In Sec. IV] we consider the case of no interactions and recover the standard results for the elastic current and noise. Our original contribution is presented in Secs. $\mathrm{V}$ and $\mathrm{VI}$, where we discuss the corrections to the current and noise due to the Hartree and the Fock diagrams, respectively. In subsection VIC we then illustrate our theory by a simple example of independent electronic levels which are coupled only via the $e$-ph interaction. Finally, we conclude and give an outlook of open issues and possible extensions of the present work in Sec. VII More technical parts of the text are deferred to 5 appendices. In addition, we make use of the Electronic Physics Auxiliary Publication Service (EPAPS) to supplement the paper with a Mathematica notebook with full expressions for the lowest order corrections to current and noise due to $e$-ph coupling. This file is intended to be of use for interested readers in order to access directly our results without need of retyping cumbersome formulas from the main text, and it also extends the results of subsection VIB to the case of finite temperature.

\section{MODEL}

The system we consider can be schematically represented as a central device region (representing the molecule or the atomic-wire) which is tunnel-coupled to non-interacting metallic leads

$$
\hat{H}=\hat{H}_{C}+\hat{H}_{L, R}+\hat{H}_{T}
$$

Neglecting for simplicity the spin degree of freedom 47 the central region can be described by the following Hamil- tonian

$$
\begin{aligned}
\hat{H}_{C} & =\hat{H}_{\mathrm{d}}+\hat{H}_{\mathrm{ph}}+\hat{H}_{e \mathrm{ph}} \\
\hat{H}_{\mathrm{d}} & =\sum_{i, j} h_{\mathrm{d}}^{i j} \hat{d}_{i}^{\dagger} \hat{d}_{j} \\
\hat{H}_{\mathrm{ph}} & =\sum_{\ell} \hbar \omega_{\ell} \hat{b}_{\ell}^{\dagger} \hat{b}_{\ell} \\
\hat{H}_{e \mathrm{ph}} & =\sum_{\ell} \sum_{i, j} M_{\ell}^{i j} \hat{d}_{i}^{\dagger} \hat{d}_{j}\left(\hat{b}_{\ell}^{\dagger}+\hat{b}_{\ell}\right),
\end{aligned}
$$

where $\hat{d}_{i}\left(\hat{d}_{i}^{\dagger}\right)$ and $\hat{b}_{\ell}\left(\hat{b}_{\ell}^{\dagger}\right)$ are the electron and phonon annihilation (creation) operators, respectively; $\hat{H}_{\mathrm{d}}$ is the single-particle effective Hamiltonian of the electrons moving in a static arrangement of atomic nuclei, $\hat{H}_{\mathrm{ph}}$ is the Hamiltonian of free uncoupled phonons, $\hat{H}_{e p h}$ is the $e$ ph coupling within the harmonic approximation, and $\mathbf{M}_{\ell}=\left\{M_{\ell}^{i j}\right\}$ is the $e$-ph coupling matrix for the $\ell$-th phonon mode. Here, boldface notation stands for matrices in the system electronic space. The leads and tunneling Hamiltonians are given by

$$
\begin{aligned}
\hat{H}_{L, R} & =\sum_{k, \alpha=L, R} \varepsilon_{\alpha, k} \hat{c}_{\alpha, k}^{\dagger} \hat{c}_{\alpha, k}, \\
\hat{H}_{T} & =\sum_{k, \alpha=L, R} \sum_{i}\left(V_{\alpha, k}^{i} \hat{c}_{\alpha, k}^{\dagger} \hat{d}_{i}+\text { h.c. }\right) .
\end{aligned}
$$

The states in the leads are occupied according to the Fermi distributions $f_{\alpha}(\varepsilon)=f\left(\varepsilon-\mu_{\alpha}\right)$, with $f(\varepsilon)=(1+$ $\left.e^{\beta \varepsilon}\right)^{-1}, \beta=1 / k_{B} T$ the inverse temperature, and $\mu_{\alpha}$ the chemical potential of lead- $\alpha$. The applied bias voltage is $e V=\mu_{L}-\mu_{R}$.

\section{METHODS}

\section{A. The generalized Keldysh Green's function technique}

To calculate the average current and the zerofrequency noise in the stationary regime, we employ the generalized non-equilibrium Keldysh Green's function technique ${ }^{34}$ In this approach, one is interested in finding the cumulant generating function $\mathcal{S}(\lambda)$, which in the case of charge transport is defined as

$$
e^{-\mathcal{S}(\lambda)}=\sum_{N} e^{i N \lambda} P_{t_{0}}(N)
$$

where $P_{t_{0}}(N)$ is the probability for $N$ charges to be transferred through the system during the measuring time $t_{0}$ and $\lambda$ is a continuous parameter known as counting field. Given $\mathcal{S}(\lambda)$, the cumulants $\left\langle\left\langle\delta N^{k}\right\rangle\right\rangle$ of the charge transfer distribution can be straightforwardly calculated according to the prescription

$$
\left\langle\left\langle\delta N^{k}\right\rangle\right\rangle=-\left.\frac{\partial^{k}}{\partial(i \lambda)^{k}} \mathcal{S}(\lambda)\right|_{\lambda=0} .
$$


Under the assumption that the measuring time $t_{0}$ is much longer than any correlation time of the system $\left(t_{0} \rightarrow \infty\right)$, the first two cumulants of $P_{t_{0}}(N)$ are directly proportional to the average current through the system $I$ and to the zero-frequency current noise $S$,

$$
I=e \frac{\langle\langle\delta N\rangle\rangle}{t_{0}}, \quad S=e^{2} \frac{\left\langle\left\langle\delta N^{2}\right\rangle\right\rangle}{t_{0}},
$$

which are the quantities we are primarily interested in.

The key idea for calculating the cumulant generating function for transport through a quantum system is to modify the Hamiltonian by introducing a time-dependent counting field $\lambda(t)$ and to relate $\mathcal{S}(\lambda)$ to the Keldysh Green's function of the system in the presence of $\lambda(t)$, i.e. to $G_{\lambda}^{i j}\left(t, t^{\prime}\right)=-i \hbar^{-1}\left\langle\mathcal{T}_{\mathcal{C}} \hat{d}_{i}(t) \hat{d}_{j}^{\dagger}\left(t^{\prime}\right)\right\rangle_{\lambda}$, where the evolution is due to the modified Hamiltonian ${ }^{34,43}$ One way to accomplish this is to add a time-dependent phase $\lambda(t) / 2$ to the tunneling matrix elements $V_{L, k}^{i}$,

$$
\hat{H}_{T} \rightarrow \hat{H}_{T}^{\lambda}=\sum_{k, j} V_{L, k}^{j} e^{-i \lambda(t) / 2} \hat{c}_{L, k}^{\dagger} \hat{d}_{j}+V_{R, k}^{j} \hat{c}_{R, k}^{\dagger} \hat{d}_{j}+\text { h.c. }
$$

with $\lambda(t)=\lambda \theta(t) \theta\left(t_{0}-t\right)$ on the forward branch of the Keldysh contour and $\lambda(t)=-\lambda \theta(t) \theta\left(t_{0}-t\right)$ on the backward one,$\frac{48}{r}$ where $\theta(x)$ is the Heaviside step-function.

Here we extend the result derived by Gogolin and Komnik $^{49}$ for the Anderson model to the case in which the central region has several electronic states. Generalizing the derivation of Ref. 49 to a multilevel system, we obtain the following expression for the derivative of the cumulant generating function

$$
\frac{\partial \mathcal{S}(\lambda)}{\partial \lambda}=t_{0} \int \frac{d \varepsilon}{2 \pi \hbar} \operatorname{Tr}_{K}\left\{\check{\Sigma}_{T}^{\prime}(\varepsilon) \check{\mathbf{G}}_{\lambda}(\varepsilon)\right\}
$$

where $\check{\mathbf{G}}_{\lambda}$ represents the Keldysh-Green's function of the system in Keldysh space

$$
\check{\mathbf{G}}_{\lambda}(\varepsilon)=\left(\begin{array}{ll}
\mathbf{G}_{\lambda}^{--}(\varepsilon) & \mathbf{G}_{\lambda}^{-+}(\varepsilon) \\
\mathbf{G}_{\lambda}^{+-}(\varepsilon) & \mathbf{G}_{\lambda}^{++}(\varepsilon)
\end{array}\right)
$$

$\check{\Sigma}_{T}^{\prime} \equiv \partial \check{\mathbf{\Sigma}}_{T} / \partial \lambda$, with $\check{\boldsymbol{\Sigma}}_{T}$ the self-energy due to the modified tunneling Hamiltonian $\hat{H}_{T}^{\lambda}$

$$
\check{\boldsymbol{\Sigma}}_{T}(\varepsilon)=\left(\begin{array}{cc}
i \sum_{\alpha=L, R} \boldsymbol{\Gamma}_{\alpha}\left[f_{\alpha}(\varepsilon)-1 / 2\right] & -i \boldsymbol{\Gamma}_{L} e^{i \lambda} f_{L}(\varepsilon)-i \boldsymbol{\Gamma}_{R} f_{R}(\varepsilon) \\
i \boldsymbol{\Gamma}_{L} e^{-i \lambda}\left[1-f_{L}(\varepsilon)\right]+i \boldsymbol{\Gamma}_{R}\left[1-f_{R}(\varepsilon)\right] & i \sum_{\alpha=L, R} \boldsymbol{\Gamma}_{\alpha}\left[f_{\alpha}(\varepsilon)-1 / 2\right]
\end{array}\right)
$$

and $\operatorname{Tr}_{K}$ stands for the trace over the electronic degrees of freedom and the Keldysh space, i.e. $\operatorname{Tr}_{K}\{\check{\mathbf{O}}\}=$ $\operatorname{Tr}\left\{\mathbf{O}^{--}+\mathbf{O}^{++}\right\}$, with $\operatorname{Tr}\{\cdots\}$ being the trace in the system electronic space. The check sign ` indicates matrices in the Keldysh space and the superscripts - / correspond to the forward/backward branch of the Keldyshcontour. Note that in Eq. (10) we have used the following sign convention for the elements of the Keldysh-matrix for the self-energy $\check{\boldsymbol{\Sigma}}$

$$
\check{\mathbf{\Sigma}}=\left(\begin{array}{cc}
\boldsymbol{\Sigma}^{--} & -\boldsymbol{\Sigma}^{-+} \\
-\boldsymbol{\Sigma}^{+-} & \boldsymbol{\Sigma}^{++}
\end{array}\right) \text {. }
$$

Finally, $\Gamma_{\alpha}^{i j}(\varepsilon)=2 \pi \sum_{k} V_{\alpha, k}^{i} V_{\alpha, k}^{j^{*}} \delta\left(\varepsilon-\varepsilon_{k, \alpha}\right)$ is the level broadening due to the coupling to the lead $\alpha$.

According to Eq. (8), the problem of evaluating current and noise (as well as any higher order cumulant of the charge transfer distribution) is reduced to the calculation of the system single-particle Green's function $\check{\mathbf{G}}_{\lambda}$. The latter can be obtained from the solution of the Dyson equation

$$
\check{\mathbf{G}}_{\lambda}(\varepsilon)=\check{\mathbf{g}}_{\lambda}(\varepsilon)+\check{\mathbf{g}}_{\lambda}(\varepsilon) \check{\mathbf{\Sigma}}_{e \mathrm{ph}}(\varepsilon) \check{\mathbf{G}}_{\lambda}(\varepsilon),
$$

where $\check{\Sigma}_{e p h}$ is the self-energy solely due to the $e$-ph coupling, and $\check{\mathbf{g}}_{\lambda}$ is the free Green's function of the system in the presence of the leads and of the counting field but without the $e$-ph interaction $\check{\mathbf{g}}_{\lambda}=\left(\check{\mathbf{g}}_{\mathrm{d}}^{-1}-\check{\mathbf{\Sigma}}_{T}\right)^{-1}$, with

$$
\check{\mathbf{g}}_{\mathrm{d}}(\varepsilon)=\left(\begin{array}{cc}
\varepsilon \mathbf{1}-\mathbf{h}_{\mathrm{d}} & 0 \\
0 & -\varepsilon \mathbf{1}+\mathbf{h}_{\mathrm{d}}
\end{array}\right)^{-1},
$$

the Green's function of the isolated dot. It is important to notice that $\check{\boldsymbol{\Sigma}}_{\text {eph }}$, depending on the Green's function of the system, is itself a function of the counting field $\lambda$ (see Sec. III C).

Finally, we remark in passing that for $\lambda \neq 0$ it is $\mathbf{g}_{\lambda}^{--}+\mathbf{g}_{\lambda}^{++} \neq \mathbf{g}_{\lambda}^{-+}+\mathbf{g}_{\lambda}^{+-}$, i.e. in the presence of the counting field $\lambda$, the four Keldysh Green's functions are all independent.

\section{B. Current and Noise}

Although Eq. (8) gives access to all cumulants of the charge transfer distribution through the system, in this work we will focus only on the study of the average current $I$ and the zero frequency noise $S$, which are the quantities most easily accessible from the experimental point of view.

The average current is directly obtained from Eq. (8) by setting $\lambda=0$

$$
I=i e \int \frac{d \varepsilon}{2 \pi \hbar} \operatorname{Tr}_{K}\left\{\check{\boldsymbol{\Sigma}}_{T}^{\prime}(\varepsilon) \check{\mathbf{G}}_{\lambda}(\varepsilon)\right\}_{\lambda=0}
$$

while the noise is given by

$$
\begin{aligned}
S= & e^{2} \int \frac{d \varepsilon}{2 \pi \hbar} \operatorname{Tr}_{K}\left\{\check{\boldsymbol{\Sigma}}_{T}^{\prime \prime} \check{\mathbf{G}}_{\lambda}+\check{\boldsymbol{\Sigma}}_{T}^{\prime} \check{\mathbf{G}}_{\lambda} \check{\boldsymbol{\Sigma}}_{T}^{\prime} \check{\mathbf{G}}_{\lambda}\right\}_{\lambda=0} \\
& +e^{2} \int \frac{d \varepsilon}{2 \pi \hbar} \operatorname{Tr}_{K}\left\{\check{\boldsymbol{\Sigma}}_{T}^{\prime} \check{\mathbf{G}}_{\lambda} \check{\boldsymbol{\Sigma}}_{e \mathrm{ph}}^{\prime} \check{\mathbf{G}}_{\lambda}\right\}_{\lambda=0},
\end{aligned}
$$



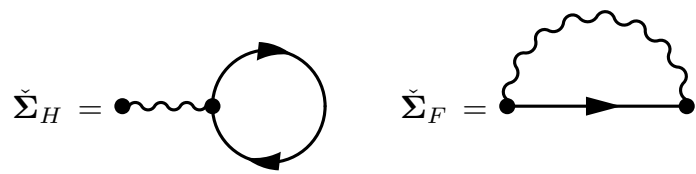

FIG. 1. Diagrammatic representations of $\check{\boldsymbol{\Sigma}}_{H}$ and $\check{\boldsymbol{\Sigma}}_{F}$. Here, the plain line stands for the free electronic Green's function $\check{\mathbf{g}}_{\lambda}$, and the wiggly line for the phononic one $\check{d}_{\ell}$. Finally, the dot represents the $e$-ph coupling constant $\mathbf{M}_{\ell}$.

where, we have used the identity $\partial_{\lambda} \check{\mathbf{O}}=-\check{\mathbf{O}}\left(\partial_{\lambda} \check{\mathbf{O}}^{-1}\right) \check{\mathbf{O}}$ together with the Dyson equation $\check{\mathbf{G}}_{\lambda}^{-1}=\check{\mathrm{g}}_{\mathrm{d}}^{-1}-\check{\boldsymbol{\Sigma}}_{T}(\lambda)-$ $\check{\Sigma}_{e p h}(\lambda)$. It turns out that the first term of Eq. (15) corresponds exactly to Eq. (30) of Ref. 50, which gives the expression for the noise within a mean-field approximation (see Appendix A). For this reason, we identify

$$
S^{(\mathrm{mf})}=e^{2} \int \frac{d \varepsilon}{2 \pi \hbar} \operatorname{Tr}_{K}\left\{\check{\boldsymbol{\Sigma}}_{T}^{\prime \prime} \check{\mathbf{G}}_{\lambda}+\check{\boldsymbol{\Sigma}}_{T}^{\prime} \check{\mathbf{G}}_{\lambda} \check{\boldsymbol{\Sigma}}_{T}^{\prime} \check{\mathbf{G}}_{\lambda}\right\}_{\lambda=0}
$$

as the mean-field contribution to noise. The remaining term constitutes the vertex correction

$$
S^{(\mathrm{vc})}=e^{2} \int \frac{d \varepsilon}{2 \pi \hbar} \operatorname{Tr}_{K}\left\{\check{\boldsymbol{\Sigma}}_{T}^{\prime} \check{\mathbf{G}}_{\lambda} \check{\mathbf{\Sigma}}_{e \mathrm{ph}}^{\prime} \check{\mathbf{G}}_{\lambda}\right\}_{\lambda=0} .
$$

As we will discuss in detail in the following, the vertex correction $S^{(\mathrm{vc})}$ can give a significant contribution to the total noise, comparable to the mean-field part and thus, contrary to what was done in some pioneering works $\stackrel{41.42}{\underline{4}}$ it cannot be omitted even in the limit of weak interaction. Moreover, neglecting $S^{(\mathrm{vc})}$ generally leads to violation of the fluctuation-dissipation theorem, see Appendix D.

\section{Weak electron-phonon coupling}

In order to make use of Eqs. (14), (15), we need to determine the full Green's function $\breve{\mathbf{G}}_{\lambda}$. Being interested in the experimentally relevant limit of weak electron-phonon coupling, we truncate the Dyson equation at the lowest (second) order in the e-ph coupling

$$
\check{\mathbf{G}}_{\lambda} \approx \check{\mathbf{g}}_{\lambda}+\check{\mathbf{g}}_{\lambda} \check{\Sigma}_{e \mathrm{ph}}^{(2)} \check{\mathbf{g}}_{\lambda},
$$

where $\check{\boldsymbol{\Sigma}}_{e \mathrm{ph}}^{(2)}=\check{\boldsymbol{\Sigma}}_{H}+\check{\boldsymbol{\Sigma}}_{F}$ is the Hartree-Fock self-energy, depicted diagrammatically in Fig. 1, with

$$
\begin{aligned}
& \boldsymbol{\Sigma}_{H}^{\eta \bar{\eta}}=\delta_{\eta \bar{\eta}} \sum_{\nu= \pm} \nu \eta \sum_{\ell} \mathbf{M}_{\ell} \operatorname{Tr}\left\{\mathbf{n}_{\lambda}^{\nu} \mathbf{M}_{\ell}\right\} d_{\ell}^{\eta \nu}(\varepsilon=0) \\
& \boldsymbol{\Sigma}_{F}^{\eta \bar{\eta}}(\varepsilon)=i \sum_{\ell} \int \frac{d \varepsilon^{\prime}}{2 \pi} d_{\ell}^{\eta \bar{\eta}}\left(\varepsilon-\varepsilon^{\prime}\right) \mathbf{M}_{\ell} \mathbf{g}_{\lambda}^{\eta \bar{\eta}}\left(\varepsilon^{\prime}\right) \mathbf{M}_{\ell},
\end{aligned}
$$

and $\eta, \bar{\eta}= \pm$. Above, $d_{\ell}^{\eta \bar{\eta}}(\varepsilon)$ represent the free thermalized phonon Green's functions of the $\ell$-th phonon mode

$$
\begin{aligned}
& d_{\ell}^{ \pm \pm}(\varepsilon)=\sum_{s= \pm}\left[-i \pi\left(2 \mathcal{N}_{\ell}+1\right) \delta\left(\varepsilon+s \hbar \omega_{\ell}\right) \pm \mathcal{P} \frac{s}{\varepsilon+s \hbar \omega_{\ell}}\right] \\
& d_{\ell}^{\mp, \pm}(\varepsilon)=-2 \pi i\left[\left(\mathcal{N}_{\ell}+1\right) \delta\left(\varepsilon \pm \hbar \omega_{\ell}\right)+\mathcal{N}_{\ell} \delta\left(\varepsilon \mp \hbar \omega_{\ell}\right)\right]
\end{aligned}
$$

with $\mathcal{N}_{\ell} \equiv\left(e^{\beta \hbar \omega_{\ell}}-1\right)^{-1}$ the thermal expectation value of the mode occupation. The proper inclusion of possible heating effects on noise, involving non-equilibrium phonon occupation and its potential back-action on the electronic transport, is beyond the scope of this publication; some of the involved issues are discussed in the concluding Sec. VII

In Eq. (19) we introduced the generalized electronic density $\mathbf{n}_{\lambda}^{\nu}$ on the two branches of the Keldysh contour $(\nu= \pm)$ in the presence of the counting field

$$
\begin{aligned}
\mathbf{n}_{\lambda}^{\nu} & \equiv \lim _{t \rightarrow t^{\prime}+0^{\nu}}-i \hbar \mathbf{g}_{\lambda}^{\nu \nu}\left(t-t^{\prime}\right) \\
& =\lim _{t \rightarrow t^{\prime}+0^{\nu}}-i \int \frac{d \varepsilon}{2 \pi} e^{-i \varepsilon\left(t-t^{\prime}\right) / \hbar} \mathbf{g}_{\lambda}^{\nu \nu}(\varepsilon) .
\end{aligned}
$$

Note that, on the two branches of the Keldysh contour, the electronic density $\mathbf{n}_{\lambda}^{\nu}$ is given by different limits $t \rightarrow t^{\prime}$ of the corresponding Green's functions $\mathbf{g}_{\lambda}^{\nu \nu}\left(t-t^{\prime}\right)$. As a consequence, even if $\lambda(t)= \pm \lambda$ on the forward/backward branch, $n_{\lambda}^{+} \neq n_{-\lambda}^{-}$for any finite value of $\lambda$. On the other hand, at $\lambda=0$ one gets

$$
\mathbf{n}_{\lambda=0}^{+}=\mathbf{n}_{\lambda=0}^{-}=\mathbf{n}_{e} \equiv-i \int \frac{d \varepsilon}{2 \pi} \mathbf{g}_{\lambda=0}^{-+}(\varepsilon),
$$

where $\mathbf{n}_{e}$ is the electronic density in the noninteracting case.

Plugging Eqs. (19), (20) into Eq. (18), we can rewrite Eqs. (14), (15) as $I=I_{\mathrm{el}}+I_{e p h}$ and $S=S_{\mathrm{el}}+S_{\text {eph }}$, where

$$
I_{\mathrm{el}}=i e \int \frac{d \varepsilon}{2 \pi \hbar} \operatorname{Tr}_{K}\left\{\check{\boldsymbol{\Sigma}}_{T}^{\prime} \check{\mathbf{g}}_{\lambda}\right\}_{\lambda=0},
$$

$$
S_{\mathrm{el}}=e^{2} \int \frac{d \varepsilon}{2 \pi \hbar} \operatorname{Tr}_{K}\left\{\check{\boldsymbol{\Sigma}}_{T}^{\prime \prime} \check{\mathbf{g}}_{\lambda}+\check{\boldsymbol{\Sigma}}_{T}^{\prime} \check{\mathbf{g}}_{\lambda} \check{\boldsymbol{\Sigma}}_{T}^{\prime} \check{\mathbf{g}}_{\lambda}\right\}_{\lambda=0},
$$

are the elastic current and noise, and

$$
I_{e \mathrm{ph}}=I_{F}+I_{H}, \quad S_{e \mathrm{ph}}=S_{F}+S_{H}
$$

give the respective corrections due to electron-phonon coupling, with

$$
I_{H(F)}=i e \int \frac{d \varepsilon}{2 \pi \hbar} \operatorname{Tr}_{K}\left\{\check{\boldsymbol{\Sigma}}_{T}^{\prime} \check{\mathbf{g}}_{\lambda} \check{\boldsymbol{\Sigma}}_{H(F)} \check{\mathbf{g}}_{\lambda}\right\}_{\lambda=0}
$$

and $S_{H(F)}=S_{H(F)}^{(\mathrm{mf})}+S_{H(F)}^{(\mathrm{vc})}$, where

$$
\begin{aligned}
S_{H(F)}^{(\mathrm{mf})}=e^{2} \int \frac{d \varepsilon}{2 \pi \hbar} \operatorname{Tr}_{K}\left\{\check{\boldsymbol{\Sigma}}_{T}^{\prime \prime} \check{\mathbf{g}}_{\lambda} \check{\boldsymbol{\Sigma}}_{H(F)} \check{\mathbf{g}}_{\lambda}\right. \\
\left.+2 \check{\boldsymbol{\Sigma}}_{T}^{\prime} \check{\mathbf{g}}_{\lambda} \check{\boldsymbol{\Sigma}}_{T}^{\prime} \check{\mathbf{g}}_{\lambda} \check{\boldsymbol{\Sigma}}_{H(F)} \check{\mathbf{g}}_{\lambda}\right\}_{\lambda=0}
\end{aligned}
$$

is the mean-field contribution and

$$
S_{H(F)}^{(\mathrm{vc})}=e^{2} \int \frac{d \varepsilon}{2 \pi \hbar} \operatorname{Tr}_{K}\left\{\check{\boldsymbol{\Sigma}}_{T}^{\prime} \check{\mathbf{g}}_{\lambda} \check{\boldsymbol{\Sigma}}_{H(F)}^{\prime} \check{\mathbf{g}}_{\lambda}\right\}_{\lambda=0} .
$$

the vertex correction. The previous equations can be schematically expressed by the diagrams of Fig. 2 


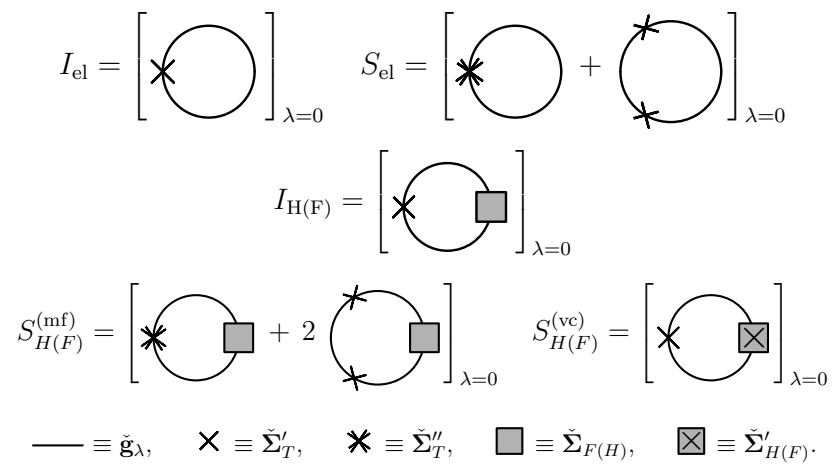

FIG. 2. Diagrammatic representations of Eqs. (23)- 26). The plain line stands for the electronic Green's function $\breve{\mathbf{g}}_{\lambda}$; single and doubled crosses stand for $\check{\boldsymbol{\Sigma}}_{T}^{\prime}$ and $\check{\boldsymbol{\Sigma}}_{T}^{\prime \prime}$, respectively. The box represents $\check{\boldsymbol{\Sigma}}_{H(F)}$ and, finally, the crossed box stands for the derivative of the $e$-ph self-energy with respect to the counting field $\check{\boldsymbol{\Sigma}}_{H(F)}^{\prime}$.

We note that truncating the Dyson equation to the lowest order in e-ph coupling, $\mathcal{O}\left(M^{2}\right)$, preserves charge conservation in that order. This implies that both mean current and zero-frequency noise are constant throughout the whole circuit, in particular at both junctions between the device and the leads. Potential violations of charge conservation can only occur in the next order, i.e. $\mathcal{O}\left(M^{4}\right)$, and can thus be safely neglected for any practical purposes in the considered weak coupling limit.

Finally, we observe that to the lowest order in the $e$ ph coupling, $I_{e \mathrm{ph}}$ and $S_{e \mathrm{ph}}$ are simply given by a linear superposition of contributions coming from different phonon modes. As a consequence, we can restrict ourselves to the case of coupling to a single phonon mode with frequency $\omega_{0}$, occupation $\mathcal{N}_{0} \equiv\left(e^{\beta \hbar \omega_{0}}-1\right)^{-1}$ and coupling matrix $\mathbf{M}$.

\section{The extended wide band limit}

The corrections to current (25) and noise (26) due to the $e$-ph coupling involve energy integrals which can be evaluated in general only numerically. Analytical progress can still be made if one assumes the electronic structure to be slowly changing over few multiples of a typical phonon energy around the Fermi level $E_{F}$ and approximate (i) the level broadening $\boldsymbol{\Gamma}_{\alpha}$ and (ii) the non-interacting retarded/advanced Green's function $\mathrm{g}^{r(a)}$ with their values at the Fermi energy $\underline{\underline{18}-20,23}$

$$
\boldsymbol{\Gamma}_{\alpha}(\varepsilon) \approx \boldsymbol{\Gamma}_{\alpha}\left(E_{F}\right) \equiv \boldsymbol{\Gamma}_{\alpha}, \quad \mathbf{g}^{r(a)}(\varepsilon) \approx \mathbf{g}^{r(a)}\left(E_{F}\right) \equiv \mathbf{g}^{r(a)},
$$

where we took $\mathbf{g}^{r(a)} \equiv \mathbf{g}_{\lambda=0}^{--}-\mathbf{g}_{\lambda=0}^{-+(+-)}$as the definition of $\mathbf{g}^{r(a)}$. This approximation, which we call "extended wide-band limit" (eWBL), is reasonable for systems where either the broadening due to tunneling is large $\left(\Gamma \gg e V, k_{B} T\right.$, and $\left.\hbar \omega_{0}\right)$, or the closest reso- nance energy $\varepsilon_{\text {res }}$ is far away from the Fermi energy $\left(\left|\varepsilon_{\text {res }}-E_{F}\right| \gg \Gamma, e V, k_{B} T\right.$, and $\left.\hbar \omega_{0}\right)$.

Within the eWBL approximation, the integration over energy of functions with compact support can be performed analytically, resulting in explicit results for the mean current and the noise as functions of the applied bias voltage and other system parameters. It should be noted however, that approximation (ii) potentially leads to problems for integrals over infinite range and, in this case, it might be necessary to lift it. Specifically, this happens in the calculation of the electron density entering the Hartree term, see Appendix [B, and in the evaluation of the real parts of the retarded/advanced Fock self-energy via Kramers-Kronig relations, see Appendix C.

\section{ELASTIC CURRENT AND NOISE}

For sake of completeness, before discussing the corrections to $I$ and $S$ due to the $e$-ph coupling, we consider briefly the results for the elastic current and noise.

In the eWBL approximation, the elastic current is simply proportional to the voltage

$$
I_{\mathrm{el}}=\frac{e}{h} \operatorname{Tr}\{\mathbf{T}\} e V
$$

with $\mathbf{T}=\boldsymbol{\Gamma}_{L} \mathbf{g}^{r} \boldsymbol{\Gamma}_{R} \mathbf{g}^{a}$, while the noise is given by

$$
S_{\mathrm{el}}=\frac{e^{2}}{h}\left[\frac{2}{\beta} \operatorname{Tr}\left\{\mathbf{T}^{2}\right\}+\operatorname{Tr}\{\mathbf{T}(\mathbf{1}-\mathbf{T})\} U(e V)\right],
$$

where we have introduced the function $U(x)=$ $x \operatorname{coth}(\beta x / 2)$.

The eigenvalues of the matrix $\mathbf{T}$ give the "PIN-code" of transmission eigen-channels of the molecule connected to leads (without e-ph interaction), and Eqs. (27), (28) are indeed equivalent ${ }^{51}$ to the standard results for current and noise in a non-interacting system derived within the scattering theory 33,52 However, $\mathbf{T}$ is not equal to the matrix product $\mathbf{t t}^{\dagger}$ of the transmission amplitudes $\mathbf{t}$ of the scattering theory (it cannot be as $\mathbf{T}$ is in general non-hermitian, for example). The two matrices are related though by a similarity (nonunitary) transformation, which among others ensures $\operatorname{Tr}\{\mathbf{T}\}=\operatorname{Tr}\left\{\mathbf{t t}^{\dagger}\right\} \stackrel{51}{=}$ With this caveat in mind, for sake of simplicity we will nevertheless call $\mathbf{T}$ the transmission matrix in the rest of this paper. The construction of the scattering eigenstates within the NGF formalism is described in detail in Ref. 53 .

We now turn our attention to the corrections to the current and noise induced by the $e$-ph interaction. In order to make the discussion as clear as possible, we will consider the contributions coming from the Hartree and the Fock diagrams separately. 


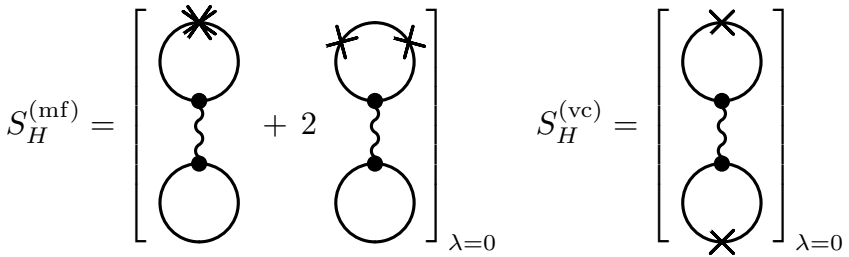

FIG. 3. Diagrammatic representations of $S_{H}^{(\mathrm{mf})}$ and $S_{H}^{(\mathrm{vc})}$. Here, plain and wiggly lines stand for the electronic and phononic Green's functions, respectively. The single cross stands for $\check{\mathbf{\Sigma}}_{T}^{\prime}$ and the doubled one for $\check{\boldsymbol{\Sigma}}_{T}^{\prime \prime}$. Finally, the dot represents the $e$-ph coupling constant $\mathbf{M}$.

\section{CORRECTIONS DUE TO THE HARTREE DIAGRAM}

\section{A. Current}

We start by considering the contributions to the current $I_{H}$ coming from the Hartree diagram. After integrating Eq. 25) in the eWBL approximation we obtain

$$
I_{H}=\frac{e}{h} \operatorname{Tr}\left\{\mathbf{T}_{H}^{(\mathrm{qel})}\right\} e V
$$

with

$$
\mathbf{T}_{H}^{(\mathrm{qel})}=-\frac{2 \operatorname{Tr}\left\{\mathbf{n}_{e} \mathbf{M}\right\}}{\hbar \omega_{0}} \boldsymbol{\Gamma}_{L}\left(\mathbf{g}^{r} \mathbf{M} \mathbf{A}_{R}+\text { h.c. }\right)
$$

with $\mathbf{n}_{e}$ the noninteracting electron density [ Eq. (22)] and $\mathbf{A}_{\alpha}=\mathbf{g}^{r} \boldsymbol{\Gamma}_{\alpha} \mathbf{g}^{a}$. The correction $I_{H}$ is therefore a smooth function of the voltage with no features at the phonon emission threshold. For this reason $I_{H}$ has been often discarded in previous works on the effects of $e$-ph interaction on the current $17-20,23,26,42,54$

It should be noticed, however, that $I_{H}$ is generally nonlinear in $e V$, since $\mathbf{n}_{e}$ can be a (smooth) function of the applied bias voltage. Such a voltage dependence is nevertheless rather weak in the eWBL (see Appendix B), and in such a case it is possible to interpret Eq. (29) as a quasi-elastic correction to an effective transmission matrix $\tilde{\mathbf{T}}=\mathbf{T}+\mathbf{T}_{H}^{(\mathrm{qel})}$, i.e. because of the e-ph coupling, the current is not proportional to the bare transmission coefficient $\operatorname{Tr}\{\mathbf{T}\}$ but rather to $\operatorname{Tr}\{\tilde{\mathbf{T}}\}$.

\section{B. Noise}

The mean-field contributions and the vertex correction to noise due to the Hartree diagram can be schematically represented by the diagrams in Fig. 3. which are the result of inserting the Hartree self-energy from Fig. 11 into appropriate diagrams in Fig. 2, In the usual eWBL, $S_{H}^{(\mathrm{mf})}$ takes the simple form

$$
\frac{S_{H}^{(\mathrm{mf})}}{e^{2} / h}=\operatorname{Tr}\left\{(\mathbf{1}-2 \mathbf{T}) \mathbf{T}_{H}^{(\mathrm{qel})}\right\} U(e V)+\frac{4}{\beta} \operatorname{Tr}\left\{\mathbf{T} \mathbf{T}_{H}^{(\mathrm{qel})}\right\}
$$

Analogously to the current $I_{H}$, this contribution has a simple interpretation in terms of the renormalization of the transmission matrix introduced above $\mathbf{T} \rightarrow \tilde{\mathbf{T}}=\mathbf{T}+$ $\mathbf{T}_{H}^{(\mathrm{qel})}$. This can be seen easily, as Eq. (31) corresponds exactly to the contribution of order $\mathbf{M}^{2}$ to the elastic shot-noise of a system with transmission matrix $\tilde{\mathbf{T}}$ :

$$
\begin{aligned}
& \frac{2}{\beta} \operatorname{Tr}\left\{\tilde{\mathbf{T}}^{2}\right\}+\operatorname{Tr}\{\tilde{\mathbf{T}}(\mathbf{1}-\tilde{\mathbf{T}})\} U(e V)=\frac{h}{e^{2}} S_{\mathrm{el}}+ \\
& +\operatorname{Tr}\left\{(\mathbf{1}-2 \mathbf{T}) \mathbf{T}_{H}^{(\mathrm{qel})}\right\} U(e V)+\frac{4}{\beta} \operatorname{Tr}\left\{\mathbf{T} \mathbf{T}_{H}^{(\mathrm{qel})}\right\}+\mathcal{O}\left(\mathbf{M}^{4}\right),
\end{aligned}
$$

where $S_{\mathrm{el}}$ is given in Eq. (28).

Making use of the cyclic invariance of the trace, the vertex correction $S_{H}^{(\mathrm{vc})}$ can be rewritten as

$$
\begin{aligned}
\frac{S_{H}^{(\mathrm{vc})}}{e^{2} / h} & =\frac{2 i}{\hbar \omega_{0}}\left[\left(\operatorname{Tr}\left\{\mathbf{M n}_{+}^{\prime}\right\}\right)^{2}-\left(\operatorname{Tr}\left\{\mathbf{M} \mathbf{n}_{-}^{\prime}\right\}\right)^{2}\right] \\
& =\frac{8}{\hbar \omega_{0}} \operatorname{Re}\left[\operatorname{Tr}\left\{\mathbf{M} \mathbf{n}_{-}^{\prime}\right\}\right] \operatorname{Im}\left[\operatorname{Tr}\left\{\mathbf{M} \mathbf{n}_{-}^{\prime}\right\}\right]
\end{aligned}
$$

where we have used the fact that $\left[\mathbf{n}_{-}^{\prime}\right]^{\dagger}=-\mathbf{n}_{+}^{\prime}$, with $\mathbf{n}_{\nu}^{\prime} \equiv\left(\partial \mathbf{n}_{\lambda}^{\nu} / \partial \lambda\right)_{\lambda=0}$. Performing the integrals over energy in the usual eWBL approximation 55 one obtains

$$
\begin{aligned}
\operatorname{Re}\left[\operatorname{Tr}\left\{\mathbf{M n}_{-}^{\prime}\right\}\right] & =-\frac{1}{2} \operatorname{Tr}\left\{\boldsymbol{\Gamma}_{L} \mathbf{A}_{R} \mathbf{M} \mathbf{g}^{a}+\text { h.c. }\right\} e V \\
\operatorname{Im}\left[\operatorname{Tr}\left\{\mathbf{M n}_{-}^{\prime}\right\}\right] & =-\frac{i}{2} \operatorname{Tr}\left\{\boldsymbol{\Gamma}_{L} \mathbf{g}_{R}^{r} \mathbf{M} \mathbf{A}_{L}-\text { h.c. }\right\} \frac{2}{\beta} \\
& -\frac{i}{2} \operatorname{Tr}\left\{\boldsymbol{\Gamma}_{L} \mathbf{g}_{R}^{r} \mathbf{M} \mathbf{A}_{R}-\text { h.c. }\right\} U(e V) \\
& +\frac{1}{4} \operatorname{Tr}\left\{\boldsymbol{\Gamma}_{L}\left(\mathbf{A}_{L} \mathbf{M} \mathbf{A}_{R}-\mathbf{A}_{R} \mathbf{M} \mathbf{A}_{R}+h . c .\right)\right\} \\
& \times\left(\frac{2}{\beta}-U(e V)\right)
\end{aligned}
$$

with $\mathbf{g}_{R}^{r}=\operatorname{Re} \mathbf{g}^{r}$. Contrary to $S_{H}^{(\mathrm{mf})}$, Eq. (32) has no simple interpretation in terms of an effective transmission coefficient and it represents a distinctive contribution to noise coming from the Hartree term. From the physical point of view, it stems from the coupling of occupations of the electronic levels with the current fluctuations. $\underline{49,56}$

We note, however, that $\operatorname{Im}\left[\operatorname{Tr}\left\{\mathbf{M n}_{-}^{\prime}\right\}\right]=0$ in the case of a system with a single electronic level symmetrically coupled to leads. Therefore in this particular case the correction to noise induced by the Hartree term is given by $S_{H}^{(\mathrm{mf})}$ alone.

\section{CORRECTIONS DUE TO THE FOCK DIAGRAM}

\section{A. Current}

We now turn our attention to the corrections to current induced by the Fock diagram. Integrating Eq. (25) in the 
usual eWBL approximation, we obtain

$$
\begin{aligned}
\frac{I_{\mathrm{F}}}{e / h} & =\operatorname{Tr}\left\{\mathbf{T}_{F}^{(\mathrm{qel})}\right\} e V+\operatorname{Tr}\left\{\mathbf{T}_{F}^{(\text {inel })}\right\} g(e V) \\
& +2 \mathcal{N}_{0} \operatorname{Tr}\left\{\mathbf{T}_{F}^{(\mathrm{qel})}+\mathbf{T}_{F}^{(\mathrm{inel})}\right\} e V+\operatorname{Tr}\left\{\mathbf{T}_{F}^{(\text {asym })}\right\} h(e V)
\end{aligned}
$$

where

$$
\begin{aligned}
& \mathbf{T}_{F}^{(\mathrm{qel})}=\boldsymbol{\Gamma}_{L}\left(\mathbf{g}^{r} \mathbf{M} \mathbf{g}_{R}^{r} \mathbf{M} \mathbf{A}_{R}+\text { h.c. }\right) \\
& \mathbf{T}_{F}^{(\mathrm{inel})}=\boldsymbol{\Gamma}_{L} \mathbf{g}^{r}\left[\mathbf{M} \mathbf{A}_{R} \mathbf{M}-\frac{i}{2}\left(\mathbf{M A M} \mathbf{g}^{r} \boldsymbol{\Gamma}_{R}-h . c\right)\right] \mathbf{g}^{a} \\
& \mathbf{T}_{F}^{(\text {asym })}=\boldsymbol{\Gamma}_{L} \mathbf{g}^{r}\left[\mathbf{M}\left(\mathbf{A}_{L}-\mathbf{A}_{R}\right) \mathbf{M g}^{r} \boldsymbol{\Gamma}_{R}+h . c .\right] \mathbf{g}^{a}
\end{aligned}
$$

with $\mathbf{A} \equiv \mathbf{A}_{L}+\mathbf{A}_{R}=i\left(\mathbf{g}^{r}-\mathbf{g}^{a}\right)$ the spectral density. All the involved quantities depend only on the properties of the system at the Fermi level and can be determined by ab-initio calculations $18-20,23$

The voltage dependence of $I_{F}$ is carried by the functions

$$
g(e V)=\frac{1}{2}\left[U\left(e V-\hbar \omega_{0}\right)-U\left(e V+\hbar \omega_{0}\right)+2 e V\right],
$$

and

$$
\begin{aligned}
h(e V) & =\frac{1}{2} \int d \varepsilon\left[\left(n_{F}(\varepsilon)-n_{F}(\varepsilon+e V)\right)\right. \\
& \left.\times \mathcal{H}_{\varepsilon^{\prime}}\left\{n_{F}\left(\varepsilon^{\prime}-\hbar \omega_{0}\right)-n_{F}\left(\varepsilon^{\prime}+\hbar \omega_{0}\right)\right\}(\varepsilon)\right],
\end{aligned}
$$

where $\mathcal{H}_{\varepsilon^{\prime}}\left\{f\left(\varepsilon^{\prime}\right)\right\}(\varepsilon)=(1 / \pi) \mathcal{P} \int d \varepsilon^{\prime} f\left(\varepsilon^{\prime}\right) /\left(\varepsilon^{\prime}-\varepsilon\right)$ is the Hilbert transform. Eq. (33) is in agreement with the result of Viljas et al., $\frac{19}{19}$ while a term $\propto(1+$ $\left.2 \mathcal{N}_{0}\right) \operatorname{Tr}\left\{\mathbf{T}_{F}^{(\mathrm{qel})}\right\} e V$ is missing in Refs. 18 and 23. Such a discrepancy is further discussed in Appendix $\mathrm{C}$.

The functions $g(e V)$ and $h(e V)$ give contributions to $d I / d V$ which are even/odd in bias, respectively (see Fig. (4). The term proportional to $h(\mathrm{eV})$ vanishes in the case of symmetric coupling to the leads, and it is typically much smaller than the contribution proportional to $g(e V)$, even for asymmetric junctions $\stackrel{23,26}{=}$ Moreover, experimentally measured conductance curves are usually very weakly asymmetric under reversal of $V$ and at present it is unclear if the asymmetry is caused by phonons or by other effects.

At low temperature $\left(k_{B} T \ll \hbar \omega_{0}, \mathcal{N}_{0} \approx 0\right)$, the main contribution to $I_{F}$ is therefore given by the first two terms of Eq. (33) alone. The first of these terms, linear in $e V$, is a quasi-elastic correction that, similarly to $I_{H}$, contributes to an effective transmission matrix $\tilde{\mathbf{T}}=\mathbf{T}+\mathbf{T}_{F}^{(\mathrm{qel})}$. The second one has instead a threshold behavior at the phonon emission energy, see Fig. 4, and it is responsible for the jump in the conductance observed in IETS and PCS experiments.

The sign of the conductance step at the phonon emission threshold (positive or negative) depends on the coefficient $\operatorname{Tr}\left\{\mathbf{T}_{F}^{(\text {inel })}\right\}$, and it has been discussed in detail in

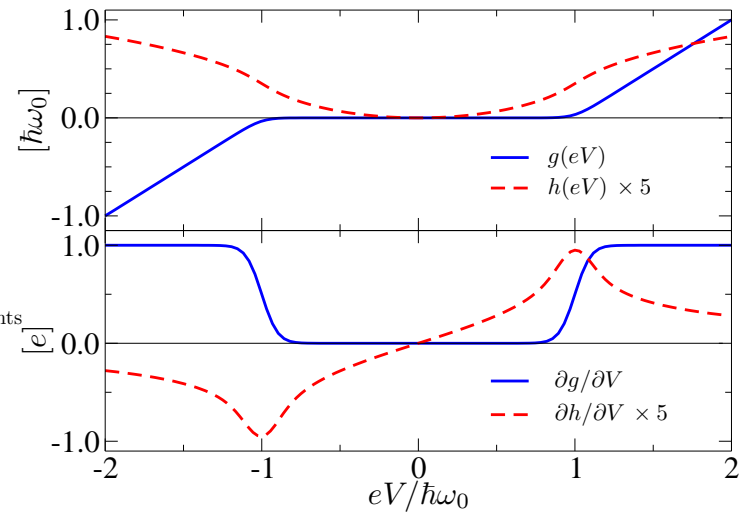

FIG. 4. Upper panel: Plots of the dependencies of the functions $g(e V)$ and $h(e V)$ on the applied bias voltage (Eqs. (35) and (36), respectively). Lower panel: Same as above, but for the derivatives $\partial g / \partial V$ and $\partial h / \partial V$. In both panels $k_{B} T=$ $\hbar \omega_{0} / 30$.

Refs. 18 20. As a rule of thumb, in the case of a molecular junction with low (high) bare transmission $\operatorname{Tr}\{\mathbf{T}\}$, inelastic $e$-ph scattering results in an increase (decrease) of the conductance above the phonon emission threshold.

In the case of a system with a single electronic level symmetrically coupled to the leads via $\Gamma_{L}=\Gamma_{R} \equiv \Gamma$, $\mathbf{T}_{F}^{(\text {inel })}$ reduces to $\mathbf{T}_{F}^{(\text {inel })}=\left(M^{2} \mathcal{T}^{2} / \Gamma^{2}\right)(1-2 \mathcal{T})$, where $\mathcal{T}=\Gamma^{2}\left|g^{r}\right|^{2}$ is the transmission coefficient. In this case, the crossover from an increase to a decrease in the conductance is predicted to occur at $\mathcal{T}=1 / 2.18,20$ This behavior has been explored and confirmed experimentally in Ref. 9.

\section{B. Noise}

We finally address the corrections to noise due to the Fock diagram $S_{F}=S_{F}^{(\mathrm{mf})}+S_{F}^{(\mathrm{vc})}$, which are schematically represented by the diagrams in Fig. 5 .

After lengthy but straightforward calculations, integration over energy in the usual eWBL approximation leads to analytic results for $S_{F}^{(\mathrm{mf} / \mathrm{vc})}$ as functions of the applied bias voltage. The final expressions are, however, rather cumbersome and, for simplicity, we consider here only the limit of zero temperature $T=0$. The complete expressions for $S_{F}^{(\mathrm{mf} / \mathrm{vc})}$ at finite temperature are given in the supplementary material,$\frac{57}{,}$ while the limit $e V \rightarrow 0$ is discussed in Appendix D in relation to the fluctuationdissipation theorem.

In the limit of zero temperature, we obtain

$$
\begin{aligned}
\frac{S_{F}^{(\mathrm{mf})}}{e^{2} / h} & =\operatorname{Tr}\left\{(\mathbf{1}-2 \mathbf{T}) \mathbf{T}_{F}^{(\mathrm{qel})}\right\}|e V| \\
& +\operatorname{Tr}\left\{(\mathbf{1}-2 \mathbf{T}) \mathbf{T}_{F}^{(\mathrm{inel})}\right\}\left(|e V|-\hbar \omega_{0}\right) \theta\left(|e V|-\hbar \omega_{0}\right) \\
& +\left.\operatorname{Tr}\left\{\mathbf{K}_{1}^{(\mathrm{mf})}\right\} \operatorname{sign}(e V) h(e V)\right|_{T=0},
\end{aligned}
$$




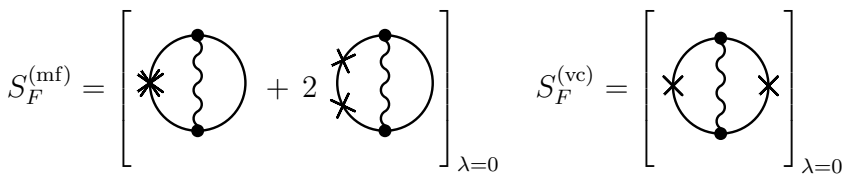

FIG. 5. Diagrammatic representations of $S_{F}^{(\mathrm{mf})}$ and $S_{F}^{(\mathrm{vc})}$. As in Fig. 3 plain and wiggly lines stand for the electronic and phononic Green's functions, respectively. The single cross stands for $\check{\mathbf{\Sigma}}_{T}^{\prime}$ and the doubled one for $\check{\boldsymbol{\Sigma}}_{T}^{\prime \prime}$. Finally, the dot represents the $e$-ph coupling constant $\mathbf{M}$.

and

$$
\begin{aligned}
\frac{S_{F}^{(\mathrm{vc})}}{e^{2} / h} & =\operatorname{Tr}\left\{\mathbf{Q}_{F}^{(\mathrm{inel})}\right\}\left(|e V|-\hbar \omega_{0}\right) \theta\left(|e V|-\hbar \omega_{0}\right) \\
& +\left.\operatorname{Tr}\left\{\mathbf{K}_{1}^{(\mathrm{vc})}\right\} \operatorname{sign}(e V) h(e V)\right|_{T=0},
\end{aligned}
$$

where $\mathbf{T}_{F}^{(\text {qel/inel) }}$ are given in Eqs. (34a), 34b),

$$
\begin{aligned}
\mathbf{Q}_{F}^{(\text {inel })}= & -\mathbf{g}^{a} \boldsymbol{\Gamma}_{L} \mathbf{g}^{r}\left[\mathbf{M} \mathbf{A}_{R} \boldsymbol{\Gamma}_{L} \mathbf{A}_{R} \mathbf{M}\right. \\
& \left.+\mathbf{M A}_{R} \boldsymbol{\Gamma}_{L} \mathbf{g}^{r} \mathbf{M} \mathbf{g}^{r} \boldsymbol{\Gamma}_{R}+\text { h.c. }\right] .
\end{aligned}
$$

and

$$
\begin{aligned}
\mathbf{K}_{1}^{(\mathrm{mf})} & =(\mathbf{1}-2 \mathbf{T}) \boldsymbol{\Gamma}_{L}\left[\mathbf{A}_{R} \mathbf{M}\left(\mathbf{A}_{L}-\mathbf{A}_{R}\right) \mathbf{M} \mathbf{g}^{a}+\text { h.c. }\right] \\
\mathbf{K}_{1}^{(\mathrm{vc})} & =\mathbf{M}\left(\mathbf{A}_{R} \boldsymbol{\Gamma}_{L} \mathbf{g}^{r}+\mathbf{g}^{a} \boldsymbol{\Gamma}_{L} \mathbf{A}_{R}\right) \mathbf{M} \\
& \times\left[\mathbf{A}_{R} \boldsymbol{\Gamma}_{L}\left(\mathbf{A}_{L}-\mathbf{A}_{R}+2 i \mathbf{g}_{R}^{r}\right)+\text { h.c. }\right] .
\end{aligned}
$$

Finally,

$$
\left.h(e V)\right|_{T=0}=\frac{\hbar \omega_{0}}{2} \sum_{s= \pm 1} s\left(\frac{e V}{\hbar \omega_{0}}+s\right) \ln \left|\frac{e V}{\hbar \omega_{0}}+s\right|
$$

is the zero temperature limit of Eq. (36). The corrections to noise $S_{F}^{(\mathrm{mf} / \mathrm{vc})}$ can then be divided into a symmetric term, which is even in bias, and an antisymmetric one, which contains the Hilbert transform $\left.h(\mathrm{eV})\right|_{T=0}$ and yields an odd contribution. We notice that, while $\left.h(\mathrm{eV})\right|_{T=0}$ is a continuous function, its derivative shows logarithmic divergencies at $\mathrm{eV}= \pm \hbar \omega_{0}$ These zero-temperature divergencies are, however, an artifact of treating the phonons as non-interacting modes, and they are regularized either by finite temperature or if any broadening of the phonon spectrum is taken into account 58,59 This issue, however, goes beyond the scope of this work.

At zero temperature, the symmetric contribution to $S_{F}$ is a piece-wise linear function of $e V$. At low voltages, $|e V|<\hbar \omega_{0}$, it is given by the first term of Eq. (37) alone. Following the same reasoning as for Eq. (31), this linear contribution can be directly interpreted in terms of the renormalization of the transmission $\mathbf{T} \rightarrow \tilde{\mathbf{T}}=\mathbf{T}+\mathbf{T}_{F}^{(\text {qel })}$, consistently with the sub-threshold correction to the current. Above the phonon emission threshold, $|e V|>\hbar \omega_{0}$, inelastic processes come into play and their contribution to the noise is given both by the second term of Eq. (37) and by the vertex correction Eq. (38). It is important to notice that these two contributions are in general of the same order (see below the Sec. VIC for a demonstrative example), so that the latter cannot be discarded.

Experimentally, $\frac{\partial S}{\partial V}$ is often measured directly by a lock-in technique. Such a quantity shows at the phonon emission threshold a sharp and distinguishable jump on top of a featureless background due to the elastic and quasi-elastic contributions. Therefore, we define here the inelastic noise signal as the difference of the plateau values of the noise derivative just above and below the jump

$$
\Delta S^{\prime}=\left.\frac{\partial S}{\partial V}\right|_{|e V|=\hbar \omega_{0}+c k_{B} T}-\left.\frac{\partial S}{\partial V}\right|_{|e V|=\hbar \omega_{0}-c k_{B} T}
$$

with $c \sim 5$ accounting for the finite jump width at finite temperatures. At low enough temperatures, terms proportional to $h(\mathrm{eV})$ give a very small contribution to the inelastic noise signal due to the symmetric shape of $\partial_{V} h$ around $|e V|=\hbar \omega_{0}$ (for details see Appendix E) and we can then approximate

$$
\Delta S^{\prime} \approx \frac{e^{3}}{h} \operatorname{Tr}\left\{(\mathbf{1}-2 \mathbf{T}) \mathbf{T}_{F}^{(\text {inel })}+\mathbf{Q}_{F}^{(\text {inel })}\right\}
$$

i.e. at low temperatures $\Delta S^{\prime}$ carries the structural information about the junction given by the terms with the threshold behavior at the phonon emission energy.

\section{Independent electronic levels}

We now consider a toy model for molecular junctions, in which we assume the electronic levels to be mutually coupled only via the $e$-ph interaction. In this case, the relevant matrices in the system electronic space are given by

$$
\left[\boldsymbol{\Gamma}_{L(R)}\right]_{i j}=\delta_{i j} \Gamma_{i, L(R)}, \quad\left[\mathbf{g}^{r}\right]_{i j}=\frac{\delta_{i j}}{\Delta_{i}+i\left(\Gamma_{i, L}+\Gamma_{i, R}\right) / 2},
$$

and $[\mathbf{M}]_{i j}=M_{i j}$, where $i, j=1, \ldots, N$, and $N$ is the number of electronic levels involved in the transport. Under the further assumption that each channel is symmetrically coupled to the leads $\left(\boldsymbol{\Gamma}_{L}=\boldsymbol{\Gamma}_{R}=\boldsymbol{\Gamma}\right)$, the prefactors $\operatorname{Tr}\left\{\mathbf{K}_{1}^{(\mathrm{mf} / \mathrm{vc})}\right\}$ vanish identically and Eqs. (37), (38) can be rewritten in a particularly suggestive form in terms of the transmission probabilities $\mathcal{T}_{i}=\Gamma_{i}^{2} /\left(\Delta_{i}^{2}+\Gamma_{i}^{2}\right)$ of the individual levels 


$$
\begin{gathered}
\frac{S_{F}^{(\mathrm{mf})}}{e^{2} / h}=2|e V| \sum_{i=1}^{N}\left\{\gamma_{i i}\left(1-\mathcal{T}_{i}\right)\left(1-2 \mathcal{T}_{i}\right)+\sum_{j>i} \gamma_{i j}\left[\mathcal{T}_{i}\left(1-2 \mathcal{T}_{i}\right)+\mathcal{T}_{j}\left(1-2 \mathcal{T}_{j}\right)\right] \sqrt{\frac{\left(1-\mathcal{T}_{i}\right)}{\mathcal{T}_{i}} \frac{\left(1-\mathcal{T}_{j}\right)}{\mathcal{T}_{j}}}\right\} \\
+\left(|e V|-\hbar \omega_{0}\right) \theta\left(|e V|-\hbar \omega_{0}\right) \sum_{i=1}^{N}\left\{\gamma_{i i}\left(1-2 \mathcal{T}_{i}\right)^{2}+2 \sum_{j>i} \gamma_{i j}\left(1-2 \mathcal{T}_{i}\left(1-\mathcal{T}_{i}\right)-2 \mathcal{T}_{j}\left(1-\mathcal{T}_{j}\right)\right)\right\}, \\
\frac{S_{F}^{(\mathrm{vc})}}{e^{2} / h}=-2\left(|e V|-\hbar \omega_{0}\right) \theta\left(|e V|-\hbar \omega_{0}\right) \sum_{i=1}^{N}\left\{2 \gamma_{i i} \mathcal{T}_{i}\left(1-\mathcal{T}_{i}\right)+\sum_{j>i} \gamma_{i j}\left[\mathcal{T}_{i}+\mathcal{T}_{j}-2 \mathcal{T}_{i} \mathcal{T}_{j}+2 \sqrt{\mathcal{T}_{i}\left(1-\mathcal{T}_{i}\right) \mathcal{T}_{j}\left(1-\mathcal{T}_{j}\right)}\right]\right\},
\end{gathered}
$$

where we have introduced the dimensionless coupling constants $\gamma_{i j}=\left|M_{i j}\right|^{2} \mathcal{T}_{i} \mathcal{T}_{j} /\left(\Gamma_{i} \Gamma_{j}\right)$. For $N=1$, Eqs. (44) reduce directly to the result of Refs. 44 46 . The voltage dependence of $S_{F}^{(\mathrm{mf})}$ and $S_{F}^{(\mathrm{vc})}$ is presented in Fig. 6 for the case of a systems with only two levels. We notice that $S_{F}^{(\mathrm{vc})}<0$ (see also Eq44b), meaning that the vertex corrections correspond to processes that lead to a suppression of the noise through the system. Moreover, Fig. [6 evidences that the contributions to the noise due to the vertex corrections can be of the same order of magnitude as the mean-field ones, and that they generally need to be taken into account in order to make accurate predictions for the phonon-assisted current noise.

In terms of the transmission coefficients of the different channels, the inelastic noise signal $\Delta S^{\prime}$ is given by

$$
\Delta S^{\prime}=\frac{e^{3}}{h} \sum_{i=1}^{N}\left\{\gamma_{i i}\left(1-8 \mathcal{T}_{i}+8 \mathcal{T}_{i}^{2}\right)+\sum_{j>i} \gamma_{i j} \varphi\left(\mathcal{T}_{i}, \mathcal{T}_{j}\right)\right\}
$$

with $\varphi\left(\mathcal{T}_{i}, \mathcal{T}_{j}\right)=2\left[\left(1-\mathcal{T}_{i}-\mathcal{T}_{j}\right)^{2}-\mathcal{T}_{i}\left(1-\mathcal{T}_{i}\right)-\mathcal{T}_{j}(1-\right.$ $\left.\left.\mathcal{T}_{j}\right)-2 \sqrt{\mathcal{T}_{i} \mathcal{T}_{j}\left(1-\mathcal{T}_{i}\right)\left(1-\mathcal{T}_{j}\right)}\right]$. Depending on the values of $\mathcal{T}_{i}$ and $\gamma_{i j}, \Delta S^{\prime}$ can be either positive or negative and it is in general very sensitive to the parameters of the junction as illustrated in Fig. 7 again for the case of a system with only two levels. Here we plot $\Delta S^{\prime}$ as a function of the transmission coefficients $\mathcal{T}_{1}, \mathcal{T}_{2}$ for different values of the $e$-ph coupling matrix elements. As general features we notice that $\Delta S^{\prime}$ is always positive when $\mathcal{T}_{1}, \mathcal{T}_{2} \ll 1$ or when they are both close to the full transmission. Vice versa, $\Delta S^{\prime}$ is always negative and close to maximum suppression for $\mathcal{T}_{1}, \mathcal{T}_{2} \approx 0.5$. Interestingly, the characteristics of $\Delta S^{\prime}$ depend strongly on the relative strength of the different matrix elements $M_{i j}$, and therefore the inelastic noise signal might provide a tool to extract important information on the $e$-ph coupling.

\section{CONCLUSIONS AND OUTLOOK}

In conclusion, in this work we have studied the corrections due to weak electron-phonon coupling to the average current and the zero-frequency noise in a molecular

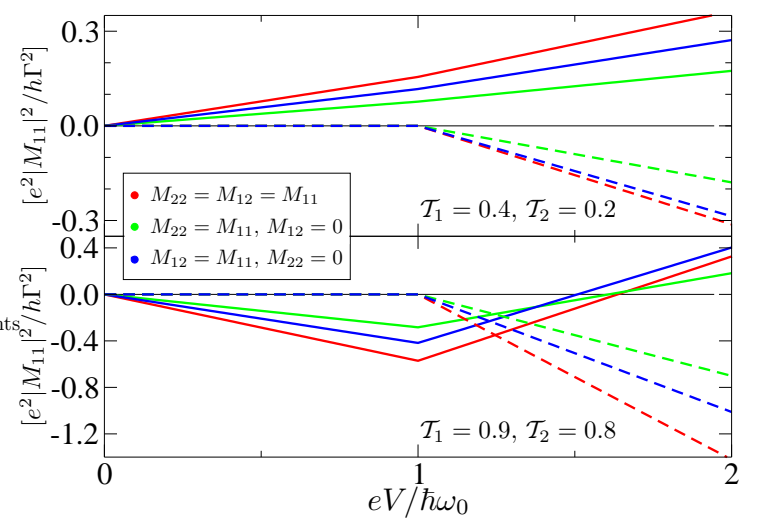

FIG. 6. (Color online) Voltage dependence of $S_{F}^{(\mathrm{mf})}$ (full lines) and $S_{F}^{(\mathrm{vc})}$ (dashed lines) for the case of the toy model (Eqs. 44a) and (44b), respectively) for different values of the transmission coefficients and of the $e$-ph coupling matrix elements. Upper panel: $\mathcal{T}_{1}=0.4, \mathcal{T}_{2}=0.2$, lower panel $\mathcal{T}_{1}=0.9, \mathcal{T}_{2}=0.8$. In both panels $k_{B} T=0$, and $\Gamma_{1}=\Gamma_{2}=\Gamma$.

junction. To address both quantities in a compact and efficient way, we employed the generalized Keldysh Green's functions technique. Importantly, for the noise we were able to identify distinct terms representing the mean-field contribution and the vertex corrections, respectively. We included in our calculations both the contributions due to the Hartree and to the Fock diagrams and, under the assumption that the densities of states of the system and of the leads depend weakly on energy (eWBL), we derived analytic expressions for $I_{H(F)}$ and $S_{H(F)}$ as functions of the applied bias voltage at arbitrary temperature. These expressions can serve as a basis for ab-initio calculations to make realistic predictions for the current noise in an experimentally significant class of molecular junctions. Finally, we considered a toy model for molecular junctions to elucidate the sensitivity of the inelastic phonon signal to the parameters characterizing the junction.

Throughout this paper we have assumed the phonon mode to be at equilibrium with an external thermal bath, i.e. we have taken the occupation $\mathcal{N}_{0}$ to be fixed according to the Bose-Einstein distribution $\mathcal{N}_{0}=\left(e^{\beta \hbar \omega_{0}}-1\right)^{-1}$. Such an approximation is strictly consistent with the 

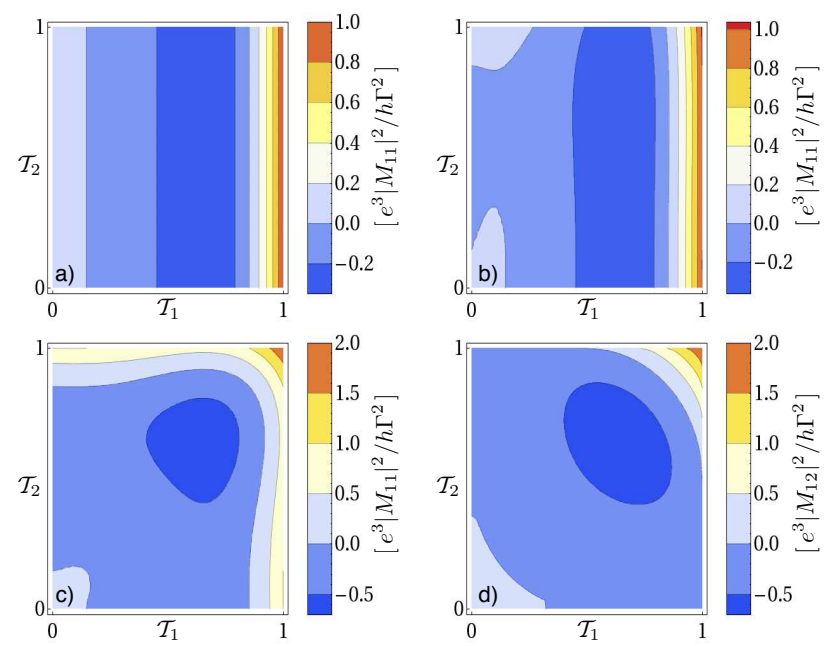

FIG. 7. (Color online) Contour plots of $\Delta S^{\prime}$ at zero temperature as a function of $\mathcal{T}_{1}$ and $\mathcal{T}_{2}$ for different values of the e-ph coupling matrix elements $M_{i j}$. a) $M_{11} \neq 0$ and $M_{22}=M_{12}=0$; b) $M_{11} \neq 0, M_{22}=0.1 M_{11}$ and $M_{12}=0$; c) $M_{11} \neq 0, M_{22}=M_{11}$ and $M_{12}=0$; d) $M_{12} \neq 0$ and $M_{11}=M_{22}=0$. In all panels, $\Gamma_{1}=\Gamma_{2}=\Gamma$.

lowest-order perturbation theory in the $e$-ph coupling when we implicitly assume strong thermalization of the phonon mode. However, it turns out in practice that often heating effects cannot be disregarded and that they influence in turn the non-linear conductance 17,19,23,39

From the theoretical point of view, the problem of nonequilibrium phonon heating can be addressed by extending the system Hamiltonian to include the coupling of the molecular phonon to other degrees of freedom (typically bulk phonons in the leads). The value of the corresponding coupling constants can ultimately be obtained from $a b$-initio calculations, which allow to asses the influence of the environment from a microscopical description $\underline{\underline{60}}$

In the case of zero counting field $\lambda=0$, the nonequilibrium phonon occupation for weak $e$-ph coupling can be equivalently obtained either by a full nonequilibrium calculation evaluating the phonon Green's function $\frac{19}{39}, 31,62$ or by solving a master equation describing the heating of the device, $\stackrel{17,23,39}{\longleftarrow}$ which can be viewed as a kinetic-equation-like approximation to the full non-equilibrium Green's functions studies. Knowing the non-equilibrium phonon occupation allows to take consistently into account the effects of phonon heating in the non-linear conductance. In our pilot study $\underline{46}$ we used such an ingredient also to phenomenologically include heating effects in the noise through a single level ${ }^{63}$

However, for a fully microscopical calculation of the noise, the situation is considerably more complicated because at finite counting field $\lambda \neq 0$, heating effects cannot be expressed solely in terms of the non-equilibrium occupation of the phonon mode. In fact, to include phonon- heating effects in the generalized Keldysh GF technique one has to solve the Dyson equation for the phonon Keldysh Green's function $\check{D}_{\lambda}=\check{d}+\check{d} \check{\Pi}_{\lambda} \check{D}_{\lambda}$, with the polarization operator $\breve{\Pi}_{\lambda}$ being given in the lowest order by the electron-hole bubble $\stackrel{19,39,64}{,}$ see Fig. 8 . Note that $\check{\Pi}_{\lambda}$ is explicitly $\lambda$-dependent via the electronic Green's functions and so is consequently also the dressed phonon Green's function $\check{D}_{\lambda}$. At $\lambda \neq 0$ the four Keldysh components of $\check{D}_{\lambda}$ are all independent and therefore, even in the kinetic limit (phonon line-width neglected), it is not possible to express the effect of heating just in terms of a single non-equilibrium occupation. It is important to notice that substituting $\check{D}_{\lambda}$ for the free phonon Green's function in the expressions for the $e$-ph self-energies $\check{\boldsymbol{\Sigma}}_{H(F)}$ generates extra (additive) contributions to the vertex corrections $S_{H}^{(\mathrm{vc})}$ and $S_{F}^{(\mathrm{vc})}$. These contributions, which are related to the influence of phonon fluctuations on the electronic transport ("feedback"), are not included in our previous phenomenological treatment of heating effects on noise $\underline{\underline{46}}$ and they could possibly account for the discrepancy between our result and an unpublished one by Jouravlev and coworkers, $\stackrel{65}{t}$ which predicts the noise to grow with voltage above the phonon emission threshold as $S_{\text {eph. }}^{\text {(Ref) }} \sim V^{4}$, in contrast to the quadratic behav-

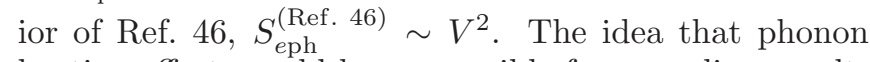
heating effects could be responsible for a nonlinear voltage dependence of $\partial S_{e p h} / \partial V$ is further corroborated by a recent work by Urban et al.,$\frac{64}{}$ which, however, predicts $S_{\text {eph }}^{\text {(Ref. 64) }} \sim V^{3}$. An independent calculation is therefore required to settle this issue. Careful inclusion of phonon heating effects into the noise calculations then certainly represents a relevant extension of our studies, furthermore urged by the relevance of heating in several IETS and PCS experiments.

Very recently, a lot of interest has been paid to the study of current-induced excitations of local spin degrees of freedom in spin-dependent IETS set-ups, $\underline{13}-15,66-70$ Several of these calculations $66,68,69$ rely on a perturbative approach analogous to the lowest order expansion of Ref. 18, also used in this paper. So far, those studies have dealt exclusively with the non-linear conductance and the study of current noise in those spin systems would be a most natural next step. Our method can be straightforwardly extended in this direction, as long as the occupation of spin states is described in a phenomenological way via the master equation 68 (or just by thermal distribution). However, possible further extensions to account for fluctuations of a non-equilibrated spin remain, even conceptually, an open question, because of the anharmonic nature of the free spin. Furthermore, the applicability of the lowest order expansion itself for the description of the spin-dependent IETS experiments seems to be currently under debate and renormalized perturbation theories might be necessary for a proper description of observed phenomena. Addressing these problems in the noise context constitutes an interesting future research direction. 


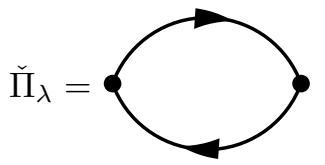

FIG. 8. Diagram corresponding to the polarization bubble for the phonon $\check{\Pi}_{\lambda}$. The plain lines and the dots represent the electronic Green's functions $\check{\mathrm{g}}_{\lambda}$ and $e$-ph coupling constant M, respectively.

Finally, the calculation of arbitrary cumulants based on the generalized Keldysh GF technique can be implemented numerically 54,64 to address the cases of structured tunneling density of states and/or stronger $e$-ph coupling, which go beyond our analytical treatment. On the other hand, for the case of weak coupling addressed in this work, such numerical methods will face convergence/efficiency problems due to very sharp phonon lineshapes and unnecessary self-consistency loops. In this respect, when complemented by ab-initio calculations for the transport coefficients $\operatorname{Tr}\{\mathbf{T}\}, \operatorname{Tr}\left\{\mathbf{T}_{H}^{(\mathrm{qel})}\right\}, \ldots$, our approach is designed to be a very efficient alternative to the full numerics in the limit of weak coupling and slowly varying electronic density of states. It uses the realistic static calculations of the electronic Green's functions, phonon modes, and their coupling as input parameters and yields reliable results for the dynamical effects in the electronic noise.

\section{ACKNOWLEDGMENTS}

We thank D. Bagrets, A.-P. Jauho, D. F. Urban, and J. M. van Ruitenbeek for useful discussions, Yu. V. Nazarov for providing us with Ref. 65, and A. Braggio for invaluable help with Mathematica. We acknowledge the financial support by DFG via SFB 767 (F. H. and W. B.), by the Czech Science Foundation via the grant 202/07/J051 and the Ministry of Education of the Czech Republic via the research plan MSM 0021620834 (T. N.).

\section{Appendix A: Mean-field contribution to noise}

Using the invariance of the trace under cyclic permutations, Eq. (16) can be recast in the following form

$$
\begin{aligned}
\frac{S^{(\mathrm{mf})}}{e^{2} / h} & =\int d \varepsilon \operatorname{Tr}\left\{i \boldsymbol{\Gamma}_{L}\left(f_{L} \mathbf{G}^{>}-\left(1-f_{L}\right) \mathbf{G}^{<}\right)\right. \\
& +\boldsymbol{\Gamma}_{L} \mathbf{G}^{>} \boldsymbol{\Gamma}_{L} \mathbf{G}^{<}+\boldsymbol{\Gamma}_{L}\left(\mathbf{G}^{r}-\mathbf{G}^{a}\right) \boldsymbol{\Gamma}_{L}\left(f_{L} \mathbf{G}^{>}\right. \\
& \left.-\left(1-f_{L}\right) \mathbf{G}^{<}\right)-f_{L}\left(1-f_{L}\right)\left(\mathbf{G}^{a} \boldsymbol{\Gamma}_{L} \mathbf{G}^{a} \boldsymbol{\Gamma}_{L}\right. \\
& \left.\left.+\mathbf{G}^{r} \boldsymbol{\Gamma}_{L} \mathbf{G}^{r} \boldsymbol{\Gamma}_{L}\right)\right\}
\end{aligned}
$$

with $\mathbf{G}^{\lessgtr} \equiv \mathbf{G}_{\lambda=0}^{\mp \pm}, \mathbf{G}^{r}=\mathbf{G}_{\lambda=0}^{--}-\mathbf{G}_{\lambda=0}^{-+}$and $\mathbf{G}^{a}=\left[\mathbf{G}^{r}\right]^{\dagger}$. Such an expression corresponds exactly ${ }^{71}$ to Eq. (30) of
Ref. 50. We stress that their result was obtained by truncating the $S$-matrix expansion by breaking two-particle Green's functions into products of one-particle Green's functions, see Refs. 50 or 72 -Sec. 13.8 for further details. This procedure holds in a mean-field theory, but it misses the contributions given by the vertex correction. For this reason we identified Eq. (16) with the mean-field contribution to noise.

It can be furthermore shown that Eq. A1 is equivalent to Eq. (10) of Ref. 41 and to the zero-frequency limit of Eq. (9) of Ref. 42, which therefore represent again solely the mean-field contribution to the noise. We stress however that, as we will discuss in Appendix D, approximating the noise with the mean-field contribution generally leads to violation of the fluctuation-dissipation theorem.

\section{Appendix B: Electronic density}

The electronic density in the system is given by

$$
\mathbf{n}_{e}=-i \int \frac{d \varepsilon}{2 \pi} \mathbf{g}_{\lambda=0}^{-+}(\varepsilon),
$$

where $\mathbf{g}_{\lambda=0}^{-+}=\left.\mathbf{g}^{r} \boldsymbol{\Sigma}_{T}^{-+} \mathbf{g}^{a}\right|_{\lambda=0}$ is the lesser Green's function without the electron-phonon coupling. It should be noticed that the integrand of Eq. (B1) does not have a finite support and therefore in this case integration over energy cannot be carried out in the eWBL approximation. Instead, the energy dependence of $\mathbf{g}^{r(a)}$ has to be taken into account while calculating the integral, and only subsequently one is allowed to consider the limits $\Gamma \gg e V, \hbar \omega_{0}, k_{B} T$ or $\left|\varepsilon_{\text {res }}-E_{F}\right| \gg \Gamma, e V, \hbar \omega_{0}, k_{B} T$ corresponding to the eWBL.

As an example we consider here the case of a system with a single electronic level symmetrically coupled to unstructured leads with constant $\Gamma_{L}=\Gamma_{R} \equiv \Gamma$. In this case

$$
n_{e}=-i \int \frac{d \varepsilon}{2 \pi} \frac{\Gamma\left[f_{L}(\varepsilon)+f_{R}(\varepsilon)\right]}{\left(\varepsilon-\varepsilon_{0}\right)^{2}+\Gamma^{2}} .
$$

Assuming zero temperature and symmetric voltage drop at the barriers $\mu_{L}=-\mu_{R}=e V / 2$ one gets

$$
\begin{aligned}
\mathbf{n}_{e} & =\frac{1}{2}+\frac{1}{2 \pi}\left[\arctan \left(\frac{e V-2 \varepsilon_{0}}{2 \Gamma}\right)-\arctan \left(\frac{e V+2 \varepsilon_{0}}{2 \Gamma}\right)\right] \\
& =\frac{1}{2}-\frac{1}{\pi} \arctan \left(\frac{\varepsilon_{0}}{\Gamma}\right)+\frac{\Gamma \varepsilon_{0}}{4 \pi\left(\Gamma^{2}+\varepsilon_{0}^{2}\right)^{2}}(e V)^{2}+\mathcal{O}\left((e V)^{3}\right),
\end{aligned}
$$

which shows that in the eWBL $\mathbf{n}_{e}$ depends very weakly on the applied bias voltage.

\section{Appendix C: Explicit form of the Fock self-energy at zero counting field}

At $\lambda=0$, the Keldysh components of $\check{\boldsymbol{\Sigma}}_{F}$ satisfy the identity $\left(\boldsymbol{\Sigma}_{F}^{--}+\boldsymbol{\Sigma}_{F}^{++}\right)_{\lambda=0}=\left(\boldsymbol{\Sigma}_{F}^{-+}+\boldsymbol{\Sigma}_{F}^{+-}\right)_{\lambda=0}$. In this 
case, it is meaningful to introduce the retarded and advanced self-energies by $\boldsymbol{\Sigma}_{F}^{r} \equiv\left(\boldsymbol{\Sigma}_{F}^{--}-\boldsymbol{\Sigma}_{F}^{-+}\right)_{\lambda=0}$ and $\boldsymbol{\Sigma}_{F}^{a}=\left[\boldsymbol{\Sigma}_{F}^{r}\right]^{\dagger}$. For definiteness, we also introduce the notation $\boldsymbol{\Sigma}_{F}^{\lessgtr} \equiv \boldsymbol{\Sigma}_{\lambda=0}^{\mp \pm}$ for the lesser and greater components at zero counting field. The latter can be easily calculated from Eq. 200 giving

$$
\begin{aligned}
\boldsymbol{\Sigma}_{F}^{<}(\varepsilon)= & i \sum_{\alpha=L, R} \mathbf{M}\left[\mathcal{N}_{0} \mathbf{A}_{\alpha}\left(\varepsilon-\hbar \omega_{0}\right) f_{\alpha}\left(\varepsilon-\hbar \omega_{0}\right)\right. \\
& \left.+\left(\mathcal{N}_{0}+1\right) \mathbf{A}_{\alpha}\left(\varepsilon+\hbar \omega_{0}\right) f_{\alpha}\left(\varepsilon+\hbar \omega_{0}\right)\right] \mathbf{M} \\
\boldsymbol{\Sigma}_{F}^{>}(\varepsilon)= & -i \sum_{\alpha=L, R} \mathbf{M}\left[\mathcal{N}_{0} \mathbf{A}_{\alpha}\left(\varepsilon+\hbar \omega_{0}\right)\left(1-f_{\alpha}\left(\varepsilon+\hbar \omega_{0}\right)\right)\right. \\
& \left.+\left(\mathcal{N}_{0}+1\right) \mathbf{A}_{\alpha}\left(\varepsilon-\hbar \omega_{0}\right)\left(1-f_{\alpha}\left(\varepsilon-\hbar \omega_{0}\right)\right)\right] \mathbf{M}
\end{aligned}
$$

The retarded self-energy can in turn be written in terms of the lesser and greater components using the identity $\boldsymbol{\Sigma}_{F}^{r}-\boldsymbol{\Sigma}_{F}^{a}=\boldsymbol{\Sigma}_{F}^{>}-\boldsymbol{\Sigma}_{F}^{<}$and Kramers-Kronig relation $\operatorname{Re} \boldsymbol{\Sigma}_{F}^{r}(\varepsilon)=\mathcal{H}_{\varepsilon^{\prime}}\left\{\operatorname{Im} \boldsymbol{\Sigma}_{F}^{r}\left(\varepsilon^{\prime}\right)\right\}(\varepsilon)$. This leads to

$$
\begin{aligned}
\operatorname{Im} \boldsymbol{\Sigma}^{r}(\varepsilon)= & -\frac{1}{2} \mathbf{M}\left\{\left(\mathcal{N}_{0}+1\right) \mathbf{A}\left(\varepsilon-\hbar \omega_{0}\right)+\mathcal{N}_{0} \mathbf{A}\left(\varepsilon+\hbar \omega_{0}\right)\right\} \mathbf{M} \\
& -\frac{1}{2} \sum_{\alpha=L, R} \mathbf{M}\left\{\mathbf{A}_{\alpha}\left(\varepsilon+\hbar \omega_{0}\right) f_{\alpha}\left(\varepsilon+\hbar \omega_{0}\right)\right. \\
& \left.-\mathbf{A}_{\alpha}\left(\varepsilon-\hbar \omega_{0}\right) f_{\alpha}\left(\varepsilon-\hbar \omega_{0}\right)\right\} \mathbf{M}
\end{aligned}
$$

$$
\begin{aligned}
\operatorname{Re} \boldsymbol{\Sigma}^{r}(\varepsilon)= & \mathbf{M}\left[\left(\mathcal{N}_{0}+1\right) \mathbf{g}_{R}^{r}\left(\varepsilon-\hbar \omega_{0}\right)+\mathcal{N}_{0} \mathbf{g}_{R}^{r}\left(\varepsilon+\hbar \omega_{0}\right)\right] \mathbf{M} \\
& -\frac{1}{2} \sum_{\alpha=L, R} \mathbf{M}\left[\mathcal{H}_{\varepsilon^{\prime}}\left\{\mathbf{A}_{\alpha}\left(\varepsilon^{\prime}\right) f_{\alpha}\left(\varepsilon^{\prime}\right)\right\}\left(\varepsilon+\hbar \omega_{0}\right)\right. \\
& \left.-\mathcal{H}_{\varepsilon^{\prime}}\left\{\mathbf{A}_{\alpha}\left(\varepsilon^{\prime}\right) f_{\alpha}\left(\varepsilon^{\prime}\right)\right\}\left(\varepsilon-\hbar \omega_{0}\right)\right] \mathbf{M},
\end{aligned}
$$

where we have used the identity $\mathcal{H}_{\varepsilon^{\prime}}\left\{\mathbf{A}\left(\varepsilon^{\prime}\right)\right\}(\varepsilon)=$ $-2 \mathcal{H}_{\varepsilon^{\prime}}\left\{\mathbf{g}_{I}^{r}\left(\varepsilon^{\prime}\right)\right\}(\varepsilon)=-2 \mathbf{g}_{R}^{r}(\varepsilon)$, with $\mathbf{g}_{R(I)}^{r}$ the real (imaginary) part of $\mathbf{g}^{r}$. We point out that the energy dependence of $\mathbf{g}^{r(a)}$ cannot be disregarded while using Kramers-Kronig relations, as the Hilbert transform $\mathcal{H}_{\varepsilon^{\prime}}$ generally involves integrals over infinitely extended range. However, in the limits $\Gamma \gg e V, \hbar \omega_{0}, k_{B} T$ or $\left|\varepsilon_{\text {res }}-E_{F}\right| \gg \Gamma, e V, \hbar \omega_{0}, k_{B} T$ corresponding to the eWBL approximation the previous expressions take a simpler form

$$
\begin{aligned}
& \operatorname{Im} \boldsymbol{\Sigma}_{F}^{r}(\varepsilon)=-\frac{1}{2} \sum_{\alpha=L, R} \mathbf{M A}_{\alpha} \mathbf{M}\left[\left(2 \mathcal{N}_{0}+1\right)\right. \\
&\left.+f_{\alpha}\left(\varepsilon+\hbar \omega_{0}\right)-f_{\alpha}\left(\varepsilon-\hbar \omega_{0}\right)\right] \\
& \operatorname{Re} \boldsymbol{\Sigma}_{F}^{r}(\varepsilon)=\left(2 \mathcal{N}_{0}+1\right) \mathbf{M g}_{R}^{r} \mathbf{M}-\frac{1}{2} \sum_{\alpha=L, R} \mathbf{M} \mathbf{A}_{\alpha} \mathbf{M} \\
& \times \mathcal{H}_{\varepsilon^{\prime}}\left\{f_{\alpha}\left(\varepsilon^{\prime}+\hbar \omega_{0}\right)-f_{\alpha}\left(\varepsilon^{\prime}-\hbar \omega_{0}\right)\right\}(\varepsilon) .
\end{aligned}
$$

Note that the Hilbert transform now involves only a function with finite support. Inserting these expressions into Eq. (25), it is easy to show that the first term of Eq. (C2) is the origin of the discrepancy between our result for $I_{F}$, Eq. (33), and the expression derived by Paulsson and coworkers, ${ }^{18,23}$ This discrepancy stems from the subtleties in the use of the eWBL mentioned above, and was already pointed out by Viljas et al. 19 whose result agrees with ours.

\section{Appendix D: Fluctuation-dissipation theorem}

The fluctuation-dissipation theorem relates the noise at zero voltage to the linear conductance of the system G

$$
S(V=0)=\frac{2}{\beta} G
$$

In the case of the contributions due to the Hartree term, it follows form Eqs. (32), (31) that $S_{H}^{(\mathrm{vc})} \rightarrow$ 0 at zero voltage, while $S_{H}^{(\mathrm{mf})}$ fulfills the fluctuationdissipation theorem

$$
S_{H}(V=0)=S_{H}^{(\mathrm{mf})}(V=0)=\frac{2}{\beta} G_{H},
$$

with $G_{H}=e^{2} / h \operatorname{Tr}\left\{\mathbf{T}_{H}^{(\mathrm{qel})}\right\}$.

The situation is, however, different for the Fock term. In fact, in the limit $e V \rightarrow 0$, both the mean-field contribution $S_{F}^{(\mathrm{mf})}$ and the vertex corrections $S_{F}^{(\mathrm{vc})}$ are different from zero and reduce to

$$
\begin{aligned}
\frac{S_{F}^{(\mathrm{mf})}(V=0)}{e^{2} / h} & =\operatorname{Tr}\left\{\mathbf{T}_{F}^{(\mathrm{qel})}\right\} \frac{2}{\beta} \frac{U\left(\hbar \omega_{0}\right)}{\hbar \omega_{0}}+\frac{U\left(\hbar \omega_{0}\right)^{2}-\left(\hbar \omega_{0}\right)^{2}}{\hbar \omega_{0}} \\
& \times \operatorname{Tr}\left\{\mathbf{T}_{F}^{(\mathrm{inel})}+\boldsymbol{\Gamma}_{L} \mathbf{g}^{r} \mathbf{M} \mathbf{A}_{L} \mathbf{M g}^{a}\right\} \\
\frac{S_{F}^{(\mathrm{vc})}(V=0)}{e^{2} / h} & =-\operatorname{Tr}\left\{\boldsymbol{\Gamma}_{L} \mathbf{g}^{r} \mathbf{M} \mathbf{A}_{L} \mathbf{M} \mathbf{g}^{a}\right\} \frac{U\left(\hbar \omega_{0}\right)^{2}-\left(\hbar \omega_{0}\right)^{2}}{\hbar \omega_{0}} .
\end{aligned}
$$

On the other hand, the correction to the linear conductance due to $\check{\Sigma}_{F}$ is given by

$$
\frac{G_{F}}{e^{2} / h}=\operatorname{Tr}\left\{\mathbf{T}_{F}^{(\mathrm{qel})}\right\} \frac{U\left(\hbar \omega_{0}\right)}{\hbar \omega_{0}}+\operatorname{Tr}\left\{\mathbf{T}_{F}^{(\mathrm{inel})}\right\} \frac{\beta}{2} \frac{U\left(\hbar \omega_{0}\right)^{2}-\left(\hbar \omega_{0}\right)^{2}}{\hbar \omega_{0}}
$$

Comparing the previous expressions one can see that

$$
S_{F}^{(\mathrm{mf})}(V=0)+S_{F}^{(\mathrm{vc})}(V=0)=\frac{2}{\beta} G_{F}
$$

but the mean field contribution alone does not satisfy the fluctuation dissipation theorem $S_{F}^{(\mathrm{mf})}(V=0) \neq 2 G_{F} / \beta$. This clearly shows that in general, even in the limit of weak e-ph coupling, vertex corrections must be included into the noise calculation in order to obtain consistent results. 


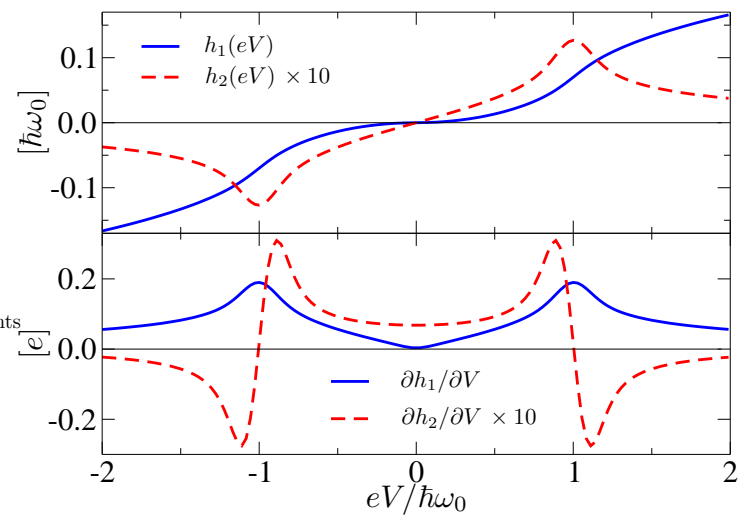

FIG. 9. Upper panel: Plots of the dependencies of the functions $h_{1}(\mathrm{eV})$ and $h_{2}(\mathrm{eV})$ on the applied bias voltage (Eqs. E3) and (E4), respectively). Lower panel: Same as above, but for the derivatives $\partial h_{1} / \partial V$ and $\partial h_{2} / \partial V$. In both panels $k_{B} T=\hbar \omega_{0} / 30$.

\section{Appendix E: Anti-symmetric contribution to $S_{F}$}

In this appendix we give the complete expression for the terms of $S_{F}^{(\mathrm{mf} / \mathrm{vc})}$ which are anti-symmetric with respect to the bias voltage. At finite temperature they are given by

$$
\begin{gathered}
\frac{S_{\mathrm{asym}}^{(\mathrm{mf})}}{e^{2} / h}=\operatorname{Tr}\left\{\mathbf{K}_{1}^{(\mathrm{mf})}\right\} h_{1}(e V)+\operatorname{Tr}\left\{\mathbf{K}_{2}^{(\mathrm{mf})}\right\} h_{2}(e V), \\
\frac{S_{\mathrm{asym}}^{(\mathrm{vc})}}{e^{2} / h}=\operatorname{Tr}\left\{\mathbf{K}_{1}^{(\mathrm{vc})}\right\} h_{1}(e V)+\operatorname{Tr}\left\{\mathbf{K}_{2}^{(\mathrm{vc})}\right\} h_{2}(e V),
\end{gathered}
$$

where $\mathbf{K}_{1}^{(\mathrm{mf} / \mathrm{vc})}$ are those of Eqs. (40) and

$$
\begin{aligned}
\mathbf{K}_{2}^{(\mathrm{mf})} & =\boldsymbol{\Gamma}_{L} \mathbf{A}_{R} \boldsymbol{\Gamma}_{L}\left[\mathbf{A}_{R} \mathbf{M}\left(\mathbf{A}_{L}-\mathbf{A}_{R}\right) \mathbf{g}^{a}+\text { h.c. }\right], \quad(\mathrm{E} 2 \mathrm{a}) \\
\mathbf{K}_{2}^{(\mathrm{vc})} & =i \mathbf{M}\left(\mathbf{A}_{R} \boldsymbol{\Gamma}_{L} \mathbf{g}^{r}+\text { h.c. }\right) \mathbf{M}\left(\mathbf{A} \boldsymbol{\Gamma}_{L} \mathbf{g}_{R}^{r}-\text { h.c. }\right)-\mathbf{K}_{1}^{(\mathrm{vc})} / 2 .
\end{aligned}
$$

The line shape of $S_{\mathrm{asym}}^{(\mathrm{mf} / \mathrm{vc})}$ is defined by the functions

$$
h_{1}(e V)=h(e V) \operatorname{coth}(\beta e V / 2) \text {, }
$$

with $h(\mathrm{eV})$ given in Eq. (36) and

$$
\begin{aligned}
h_{2}(e V)= & \int d \varepsilon\left[n_{F}(\varepsilon+e V)\left[1-n_{F}(\varepsilon+e V)\right]\right. \\
& \left.\times \mathcal{H}_{\varepsilon^{\prime}}\left\{n_{F}\left(\varepsilon^{\prime}-\hbar \omega_{0}\right)-n_{F}\left(\varepsilon^{\prime}+\hbar \omega_{0}\right)\right\}(\varepsilon)\right] .
\end{aligned}
$$

We notice that $h_{2}(e V)=(e \beta)^{-1} \partial_{V} h(e V)$, i.e. $h_{2}(e V)$ is directly proportional to the derivative of $h(\mathrm{eV})$ but it exhibits no divergencies and actually tends to zero in the limit $T \rightarrow 0$ due to the suppression factor $1 / \beta$ with respect to $h(\mathrm{eV})$. Furthermore, we observe once again that $S_{\mathrm{asym}}^{(\mathrm{mf} / \mathrm{vc})}$ give a negligible contribution to the inelastic noise signal $\Delta S^{\prime}$ at low temperatures, since $\partial_{V} h_{1}$ is almost symmetric around the phonon emission threshold, i.e. $\left.\left.\partial_{V} h_{1}\right|_{|e V| \gtrsim \hbar \omega_{0}} \approx \partial_{V} h_{1}\right|_{|e V| \lesssim \hbar \omega_{0}}$, and $\partial_{V} h_{2}$ is suppressed by low temperature, see Fig. 9
* federica.haupt@uni-konstanz.de

† tno@karlov.mff.cuni.cz

¥ wolfgang.belzig@uni-konstanz.de

1 G. Cuniberti, G. Fagas, and K. Richter, eds., Introducing Molecular Electronics (Springer, Berlin, 2005).

2 N. Agraït, A. L. Yeyati, and J. M. van Ruitenbeek, Phys. R ep., 377, 81 (2003), ISSN 0370-1573.

3 Y. G. Naidyuk and I. K. Yanson, Point-Contact Spectroscopy (Springer, Berlin, 2005).

4 R. C. Jaklevic and J. Lambe, Phys. Rev. Lett., 17, 1139 (966).

5 B. C. Stipe, M. A. Rezaei, and W. Ho, Science, 280, 1732 (998)

6 . Agraï, C. Untiedt, G. Rubio-Bollinger, and S. Vieira, Phys. Rev. Lett., 88, 216803 (2002).

7 R. H. M. Smit, Y. Noat, C. Untiedt, N. D. Lang. M. C. van Hemert, and J. M. van Ruitenbeek, Nature, 419, 906 (\$002).

8 D. Djukic, K. S. Thygesen, C. Untiedt, R. H. M. Smit, K. W. Jacobsen, and J. M. van Ruitenbeek, Phys. Rev. $\mathrm{B}, \mathbf{7 1 , 1 6 1 4 0 2 ( 2 0 0 5 )}$

9 O. Tal, M. Krieger, B. Leerink, and J. M. van Ruitenbeek, Phys. Rev. Lett., 100, 196804 (2008).
10 T. Frederiksen, K. J. Franke, A. Arnau, G. Schulze, J. I. Pascual, and N. Lorente, Phys. Rev. B, 78, 233401 (2008).

11 M. Rahimi and M. Hegg, Phys. Rev. B, 79, 081404 (2009).

12 C. R. Arroyo, T. Frederiksen, G. Rubio-Bollinger, M. Vélez, A. Arnau, D. Sánchez-Portal, and N. Agraït, Phys. Rev. B, 81, 075405 (2010).

13 A. J. Heinrich, J. A. Gupta, C. P. Lutz, and D. M. Eigler, Science, 306, 466 (2004),

14 C. F. Hirjibehedin, C. P. Lutz, and A. J. Heinrich, Science, 312, 1021 (2006).

${ }^{15}$ Y.-S. Fu, T. Zhang, S.-H. Ji, X. Chen, X.-C. Ma, J.-F. Jia, and Q.-K. Xue, Phys. Rev. Lett.. 103. 257202 (2009).

16 N. Lorente and M. Persson, Phys. Rev. Lett., 85, 2997 (2000).

T. Frederiksen, M. Brandbyge, N. Lorente, and A.-P. Jauho, Phys. Rev. Lett., 93, 256601 (2004).

18 M. Paulsson, T. Frederiksen, and M. Brandbyge, Phys. Rev. B, 72, 201101 (2005).

${ }^{19}$ J. K. Viljas, J. C. Cuevas, F. Pauly, and M. Häfner, Phys. Rev. B, 72, 245415 (2005).

L. de la Vega, A. Martín-Rodero, N. Agraït, and A. L. Yeyati, Phys. Rev. B, 73, 075428 (2006). 
21 G. C. Solomon, A. Gagliardi, A. Pecchia, T. Frauenheim, A. D. Carlo, J. R. Reimers, and N. S. Hush, J. Chem. Phys., 124, 094704 (2006).

22 N. Sergueev, A. A. Demkov, and H. Guo, Phys. Rev. B. 7 5, $233418(2007)$.

T. Frederiksen, M. Paulsson, M. Brandbyge, and A.-P. ${ }^{50}$ F. M. Souza, A. P. Jauho, and J. C. Egues, Phys. Rev. B, J euho, Phys. Rev. B, 75, 205413 (2007). 78, 155303 (2008).

24 G. Teobaldi, M. Peñalba, A. Arnau, N. Lorente, and W. A. ${ }^{11}$ Y. Meir and N. S. Wingreen, Phys. Rev. Lett., 68, 2512 Hofer, Phys. Rev. B, 76, 235407 (2007), (1992).

25 T. Frederiksen, N. Lorente, M. Paulsson, and M. Brand- ${ }^{52}$ M. Büttiker, Phys. Rev. B, 46, 12485 (1992) byge, Phys. Rev. B, 75, 235441 (2007).

26 M. Paulsson, T. Frederiksen, H. Ueba, N. Lorente, and Prandbye Phys Rey Lett. 100, 226604 (2008) and

27 I. S. Kristensen, M. Paulsson, K. S. Thygesen, and K. W.

Jacobsen, Phys. Rev. B, 79, 235411 (2009)

28 M. Alducin. D. Sánchez-Portal, A. Arnau, and N. Lorente, Phys. Rev. Lett., 104, 136101 (2010).

29 J. Fransson, H. C. Manoharan, and A. V. Balatsky, Nand Lett., 10, 1600 (2010).

30 S. Monturet, M. Alducin, and N. Lorente, Phys. Rev. B, $82,085447(2010)$.

31 K. R. Patton, arXiv:1007.1238v1 (2010) (unpublished)

32 q. Beenakker and C. Schönenberger, Physics Today, 56. 3 (2003)

33 Y. M. Blanter and M. Büttiker, Phys. Rep., 336, 1 (2000).

34 Y. V. Nazarov, Ann. Phys., 8, SI-193 (1999); Y. V Nazarov, ed., Quantum Noise in Mesoscopic Physics (\$pringer, Berlin, 2003).

35 H. E. van den Brom and J. M. van Ruitenbeek, Phys. Rev. Lett., 82, 1526 (1999).

${ }^{36}$ D. Djukic and J. M. van Ruitenbeek, Nano Lett., 6, 789 (1006).

37 M. Kıguchi, O. Tal, S. Wohlthat, F. Pauly, M. Krieger. D. Djukic, J. C. Cuevas, and J. M. van Ruitenbeek, Phys. R ev. Lett., 101, 046801 (2008).

$38 \mathrm{~J}$ M. van Ruitenbeek, private communication.

39 A. Mitra, 1. Aleiner, and A. J. Millis, Phys. Rev. B, 69, $245302(2004)$

40 J Koch and F. von Oppen, Phys. Rev. Lett., 94, 206804 (005): J. Koch, M. E. Raikh, and F. von Oppen, ibıd., 9 5, $056801(2005)$.

41 J.-X. Zhu and A. V. Balatsky, Phys. Rev. B, 67, 165326 (\$003).

42 M. Galperin, A. Nitzan, and M. A. Ratner, Phys. Rev. B, 74,075326 (2006).

43 L S. Levitov and G. B. Lesovik, JETP Lett., 58, 230 (1993); L. S. Levitov, H. W. Lee, and G. B. Lesovik, J. Math. Phys., 37, 4845 (1996)

44 T. L. Schmidt and A. Komnik, Phys. Rev. B, 80, 041307 (2009)

45 R. Avriller and A. Levy Yeyati, Phys. Rev. B, 80, 041309 (009).

46 F. Haupt, T. Novotný, and W. Belzig, Phys. Rev. Lett., 1p3, 136601 (2009)

47 Based on a spin-less model, our results need to be multiplied by a factor of 2 when compared with works where spin degeneracy is explicitly taken into account.

L. S. Levitov and M. Reznikov, Phys. Rev. B, 70, 115305 (2004).

53 M. Paulsson and M. Brandbyge, Phys. Rev. B, 76, 115117 (2007).

J. Fransson and M. Galperin, Phys. Rev. B, 81, 075311 (2010)

Unlike $\mathbf{n}_{e}$, the evaluation of $\mathbf{n}_{\nu}^{\prime}$ involves only integrands with compact support.

56 S. Hershfield, Phys. Rev. B, 46, 7061 (1992).

57 See associated Mathematica notebook for the complete expression for $S_{F}^{(\mathrm{mf} / \mathrm{vc})}$ at finite temperature.

58 O. Entin-Wohlman, Y. Imry, and A. Aharony, Phys. Rev. B, 80, 035417 (2009).

R. Egger and A. O. Gogolin, Phys. Rev. B, 77, 113405 (2008)

M. Engelund. M. Brandbyge, and A. P. Jauho, Phys. Rev. B, 80, 045427 (2009).

61 Y. Asai, Phys. Rev. B, 78, 045434 (2008)

62 D. A. Ryndyk and G. Cuniberti, Phys. Rev. B, 76, 155430 (2007)

Its generalization to multilevel case is straightforward and follows exactly the lines of Refs. 17 and 23 .

${ }^{64}$ D. F. Urban, R. Avriller, and A. Levy Yeyati, Phys. Rev. B, 82, 121414 (2010).

O. N. Jouravlev, Noise and Spin in Nanostructures, Ph.D. thesis, TU Delft, Netherlands (2005).

66 J. Fernández-Rossier, Phys. Rev. Lett., 102, 256802 (2009): M. Persson, ibid., 103, 050801 (2009); J. Fransson, Nano Lett., 9, 2414 (2009)

67 N. Lorente and J.-P. Gauyacq, Phys. Rev. Lett., 103, 176601 (2009): J.-P. Gauvaca. F. D. Novaes, and N. Lorente, Phys. Rev. B, 81, 165423 (2010): F. D. Novaes, N. Lorente, and J.-P. Gauyacq, ibid., 82, 155401 (2010).

68 F. Delgado, J. J. Palacios, and J. Fernández-Rossier, Phys. Rev. Lett., 104, 026601 (2010); F. Delgado and J. Fernández-Rossier, arXiv:1006.5608v1 (2010)

69 J. Fransson, O. Eriksson, and A. V. Balatsky, Phys. Rev. B, 81, 115454 (2010).

F. Elste and C. Timm, Phys. Rev. B, 81, 024421 (2010)

71 Apart from an extra factor of 2 in Eqs. (30), (31) of Ref. 50 stemming from their different definition of the zerofrequency noise.

${ }^{72}$ H. Haug and A.-P. Jauho, Quantum Kinetics in Transport and Optics of Semiconductors, 2nd ed. (Springer, Berlin, 2008). 